\title{
Genome-wide identification of enhancers in skeletal muscle: the role of MyoD1
}

\author{
Roy Blum, ${ }^{1,2}$ Vasupradha Vethantham, ${ }^{1,2}$ Christopher Bowman, ${ }^{1,2}$ Michael Rudnicki, ${ }^{3}$ \\ and Brian D. Dynlacht ${ }^{1,2,4}$ \\ ${ }^{1}$ Department of Pathology, ${ }^{2}$ Cancer Institute, Smilow Research Center, New York University School of Medicine, New York, \\ New York 10016, USA; ${ }^{3}$ Ottawa Hospital Research Institute, Ottawa, Ontario K1H 8L6, Canada
}

To identify the compendium of distal regulatory elements that govern myogenic differentiation, we generated chromatin state maps based on histone modifications and recruitment of factors that typify enhancers in myoblasts and myotubes. We found a striking concordance between the locations of these newly defined enhancers, MyoD1-binding events, and noncoding RNA transcripts. These enhancers recruit several sequencespecific transcription factors in a spatially constrained manner around MyoD1-binding sites. Remarkably, MyoD1null myoblasts show a wholesale loss of recruitment of these factors as well as diminished monomethylation of H3K4 (H3K4me1) and acetylation of H3K27 (H3K27 ac) and reduced recruitment of Set7, an H3K4 monomethylase. Surprisingly, we found that $\mathrm{H} 3 \mathrm{~K} 4 \mathrm{me1}$, but not $\mathrm{H} 3 \mathrm{~K} 27 \mathrm{ac}$, could be restored by re-expression of $\mathrm{MyoD1}$ in $\mathrm{MyoD1}^{-/}$ myoblasts, although re-expression of this factor in MyoD1-null myotubes restored both histone modifications. Our studies identified a role for MyoD1 in condition-specific enhancer assembly through recruitment of transcription factors and histone-modifying enzymes that shape muscle differentiation.

[Keywords: ChIP-seq; chromatin modifications; epigenetics; muscle myogenesis; MyoD1; transcriptional enhancers] Supplemental material is available for this article.

Received July 24, 2012; revised version accepted October 24, 2012.

Exquisitely orchestrated gene expression programs resulting from the concerted interplay of regulatory elements at promoters and enhancers mediate differentiation and development. Although the regulation of promoters has been studied extensively, the function of distant regulatory elements remains less well understood, and it is likely that diverse mechanisms will drive enhancer function. For example, enhancers could interact with promoters through looping (Bulger and Groudine 2011) and erase Polycomb proteins and attendant trimethylation of histone H3 Lys 27 (H3K27me3) from the CpG islands of associated genes (Vernimmen et al. 2011). The observation that small RNAs (eRNAs) and large noncoding RNAs (ncRNAs) are transcribed across enhancers adds another level of regulation and suggests additional modes of enhancer function (De Santa et al. 2010; Kim et al. 2010). Although the function of eRNAs is currently unknown, they are thought to be distinct from long ncRNAs, which have been shown to regulate expression largely in trans (Cabili et al. 2011; Guttman et al. 2011).

Enhancers are associated with recruitment of RNA polymerase II (Pol II) and the histone acetyltransferase p300 as well as enrichment of several histone modifica-

${ }^{4}$ Corresponding author

E-mail brian.dynlacht@nyumc.org

Article is online at http://www.genesdev.org/cgi/doi/10.1101/gad.200113.112. tions (monomethylation of $\mathrm{H} 3 \mathrm{~K} 4$ [H3K4mel] and acetylation of H3K27 [H3K27ac]). These observations have accelerated enhancer identification and analysis, particularly in embryonic stem (ES) cells (Heintzman et al. 2007; Visel et al. 2009; for review, see Bulger and Groudine 2011). Recent studies have also begun to define enhancers as "active" or "poised" based on the combinations of chromatin marks observed in a given state. Thus, H3K27ac and $\mathrm{H} 3 \mathrm{~K} 4 \mathrm{mel}$ at distal elements correlate with genes in an active state, whereas $\mathrm{H} 3 \mathrm{~K} 4 \mathrm{me} 1$ alone or the combination of this mark with $\mathrm{H} 3 \mathrm{~K} 27 \mathrm{me} 3$ could dictate a poised or inactive state (Creyghton et al. 2010; Rada-Iglesias et al. 2011). Chromatin marks at enhancers are not static and can be dynamically regulated during differentiation. For example, H3K27me3 is replaced by H3K27ac as ES cells differentiate into neurons or as myoblasts differentiate into myotubes (Asp et al. 2011; Rada-Iglesias et al. 2011). Tissue-specific transcription factor (TF) binding is another hallmark of enhancers /Creyghton et al. 2010), and one prediction is that such factors will assist in the condition-specific recruitment of histone-modifying enzymes.

Genome-wide analyses have identified the location of MyoD1-binding events at promoters and intergenic regions in myoblasts and myotubes (Blais et al. 2005; Cao et al. 2010). Binding of MyoD1 appears to correlate with locally enhanced acetylation of histone $\mathrm{H} 4$ (Cao et al. 
2010). However, since MyoD1 was shown to bind constitutively to many sites, the extent to which these MyoD1binding sites contribute to deposition of active histone marks, enhancer function, and gene expression is not known. In addition, genome-wide studies have not yet revealed the mechanisms through which MyoD1 regulates enhancers. Here, using methods to detect enhancer signatures, we identified a compendium of regulatory elements that regulate skeletal muscle differentiation. We report a strong association between these enhancers and recruitment of MyoD1. We found that these regulatory elements are associated with recruitment of Pol II as well as ncRNAs. MyoD1 plays a pivotal, genome-wide role in recruiting ancillary sequence-specific factors, resulting in enhancer assembly and concomitant recruitment of histone-modifying enzymes (p300 and Set7) that effect deposition of $\mathrm{H} 3 \mathrm{~K} 4 \mathrm{me} 1$ and $\mathrm{H} 3 \mathrm{~K} 27 \mathrm{ac}$, marks that fail to accumulate at enhancers in $\mathrm{MyoD}^{-/-}$cells. We propose a model in which MyoD1 acts to coordinate the precise timing of specific chromatin-associated events during myogenic differentiation.

\section{Results}

Genome-wide identification of active enhancers in muscle

Our previous genome-wide studies allowed us to focus on differentiation-dependent changes in chromatin modifications over coding regions and promoters (Asp et al. 2011). We expanded these studies by investigating epigenetic modifications at transcriptional enhancers (Fig. 1A). To identify these elements genome-wide, we examined features strongly associated with distal enhancers (namely, H3K4mel and Pol II binding) (Asp et al. 2011) and performed additional chromatin immunoprecipitation (ChIP) combined with deep sequencing (ChIP-seq) experiments in C2C12 myoblasts and myotubes to map the deposition of H3K27ac and binding of p300, which acetylates H3K27 (Jin et al. 2011). In addition, we analyzed H3K18ac, as this mark is associated with p300 activity and is known to overlap with enhancers (Wang et al. 2008). In agreement with previous studies, we showed that the occurrence of p300 and H3K27ac on gene promoters strongly correlated with gene expression in both states (Fig. 1B).

To identify marked genomic regions that could function as constitutive or condition-specific enhancers of expression in muscle, we adopted several stringent criteria (Supplemental Material). This analysis resulted in a group of genomic regions that was distributed nearly equally between myoblasts and myotubes (Fig. 1C). Next, we selected a panel of genomic regions that were marked by combinations of enhancer marks in a condition-dependent manner. By employing luciferase reporter assays, we showed that each genomic region marked in myoblasts increased basal expression in myoblasts when inserted at a distance (Fig. 1D). In contrast, none of the regions marked in myotubes augmented transcription in myoblasts, indicating condition-specific function for these putative myoblast-enhancing regions and reinforcing a correlation between the deposition of these marks and potent enhancer activity.

To further confirm our ChIP-seq data, we randomly selected a subset of condition-specific enhancers that were associated with genes expressed in a condition-specific manner and validated each with quantitative ChIP (qChIP) (Fig. 1E; Supplemental Fig. S1A). To rigorously identify peaks with the hallmarks of active enhancers, we considered regions that were marked by a combination of at least three enhancer-related features and classified 5-kb regions as myoblast- or myotube-specific (uniquely marked in the indicated condition) and constitutive (marked in both conditions) enhancers (Supplemental Fig. S1B; Supplemental Table S1; Supplemental Material).

\section{Conservation and condition specificity of enhancers}

In keeping with recent studies in ES cells (Creyghton et al. 2010), bone marrow cells (De Santa et al. 2010), and cortical neurons (Kim et al. 2010), we speculated that peaks associated with $\mathrm{H} 3 \mathrm{~K} 4 \mathrm{me} 1, \mathrm{H} 3 \mathrm{~K} 27 \mathrm{ac}, \mathrm{p} 300$, and Pol II represent "active" enhancers. Since regulatory elements such as enhancers are subject to purifying selection (Siepel et al. 2005), we evaluated conservation of these regions across 30 placental mammalian genomes using phastCons scoring (Siepel et al. 2005). We found that the most highly conserved elements clustered within the central regions corresponding to the sequences associated with distinct combinations of at least three marks (Fig. 2A). To enrich for regions with the greatest regulatory potential, we pooled all peaks belonging to the four most highly conserved groups, and all subsequent analyses focused on this group of potentially active enhancers. Overall, we identified 4315 and 6313 potential enhancers in myoblasts and myotubes, respectively. Among this group, 2844 and 4826 were marked in a condition-specific manner in myoblasts and myotubes, respectively, whereas the remaining enhancers were constitutively marked in both conditions (Fig. 1A; Supplemental Table S1).

To further determine the degree of sequence conservation, we compared our potential enhancers with a published set of nearly 172,000 noncoding sequences that are highly conserved between humans and rodents (Prabhakar et al. 2006). We found that $34 \%$ of myoblast enhancers and $36 \%$ of myotube enhancers overlapped conserved noncoding sequences (CNSs) (Fig. 2B; Supplemental Material). Association of condition-specific enhancers with CNSs was statistically significant for CNSs with cutoff values higher than a $P$-value $\leq 10^{-20}$. However, no statistical significance was found when elements with an extreme degree of evolutionary conservation, mostly corresponding to developmental genes (Visel et al. 2008), were considered. Therefore, muscle-specific enhancers are strongly conserved but not ultraconserved, consistent with other tissue-specific elements whose evolution occurs at higher rates (Ponting 2008).

We performed several studies to determine whether our compendium overlapped with previously described regulatory elements. First, we took advantage of the VISTA enhancer project-a well-curated database of 
A
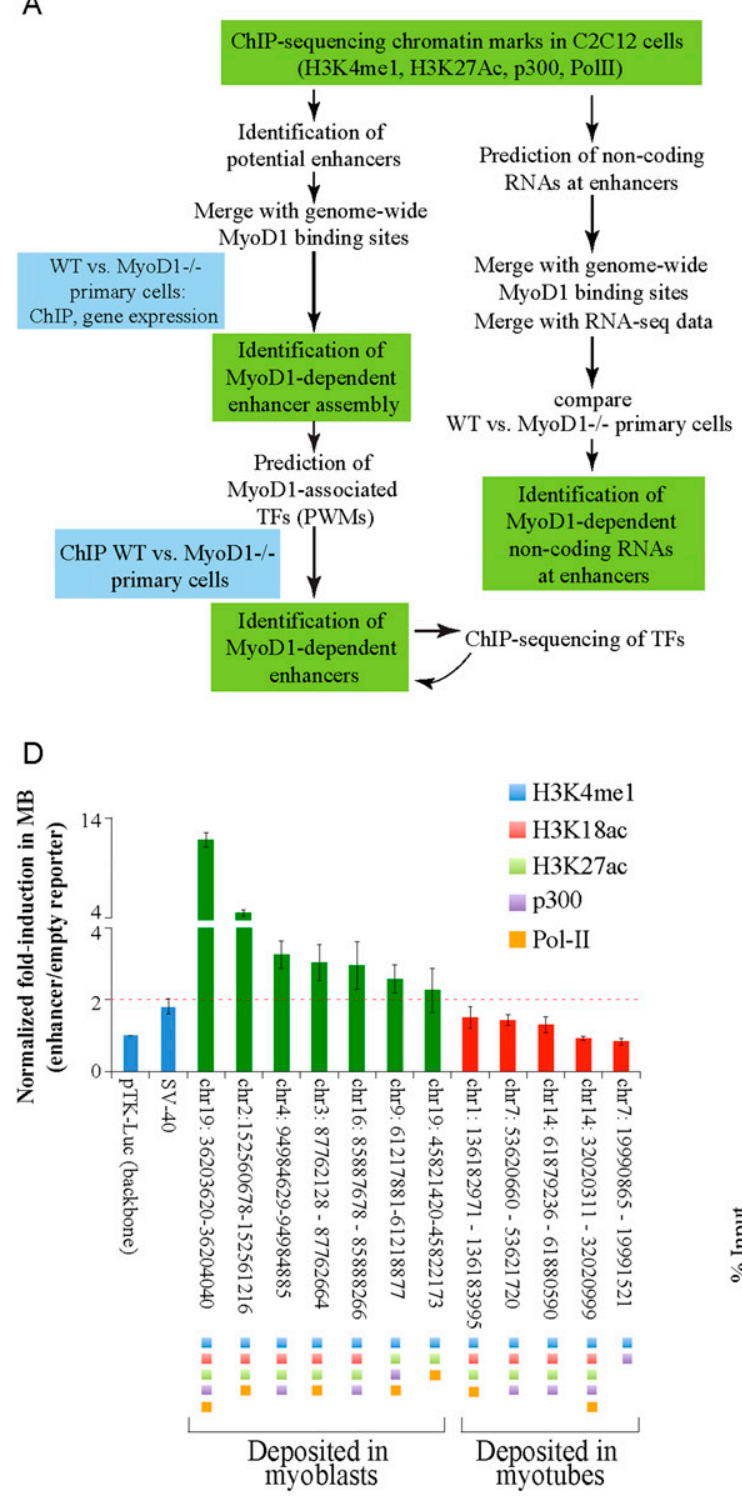

B

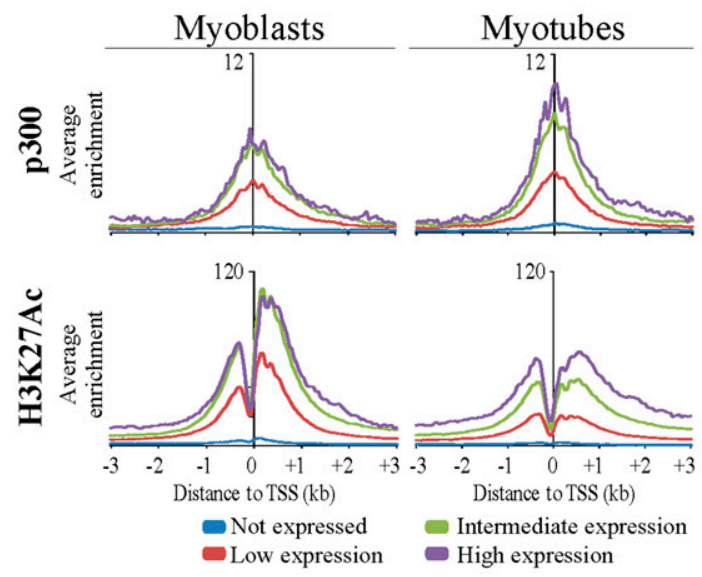

C

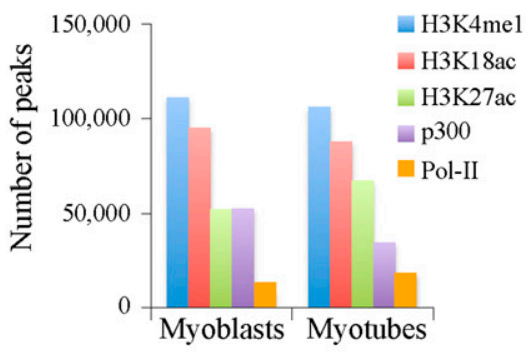

E

H3K27ac ChIP

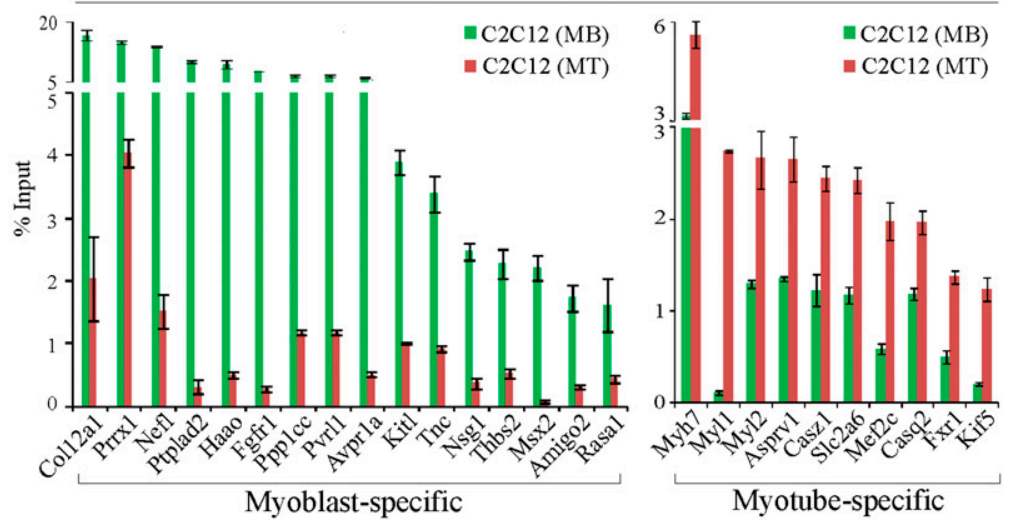

Figure 1. Genome-wide identification of muscle enhancers. $(A)$ Approach used to elucidate myogenic enhancers. $(B)$ ChIP-seq analyses of p300 and H3K27ac indicate strong correlations with gene expression levels. The average ChIP-seq enrichment per 50-base-pair (bp) bin for the total population of genes in the four expression groups (see the Supplemental Material) was plotted with respect to the TSSs of coding genes. The $Y$-axis presents the average $\log 2$ enrichment value. $(C)$ Distribution of enhancer-related peaks (triply marked) in myoblasts and myotubes. Peaks that satisfy the criteria for enhancer identification are depicted quantitatively as described in the Materials and Methods. $(D)$ A series of putative enhancer regions were tested using a luciferase reporter assay. Genomic regions were marked by the indicated enhancer-related features. (E) qChIP was used to validate H3K27ac deposition at condition-specific enhancers associated with genes that were highly expressed in a condition-dependent manner. Enriched DNA was analyzed by quantitative PCR (qPCR). ChIP enrichment is shown as percent of input. Error bars depict the standard error of the mean (SEM) derived from three independent experiments. (MB) Myoblasts; (MT) myotubes.

distant-acting enhancers identified based on evolutionarily conserved noncoding sequences (Visel et al. 2007). We discovered 34 muscle enhancers that were also reported to be active enhancers in mice, and this correspondence was statistically significant when compared with random genomic sets (Supplemental Fig. S1C; Supplemental Table S2). In addition, consistent with reports of verified skeletal muscle enhancers, Myl1, Myod1, and Sgcg distal enhancers showed prominent enhancer signatures in myotubes (Supplemental Fig. S1D).

Interestingly, we found that although each of the individual enhancer-related marks was equally enriched in myoblasts and myotubes, the ratio of enhancers was, on average, twofold higher in myotubes versus myoblasts 
Blum et al.

A
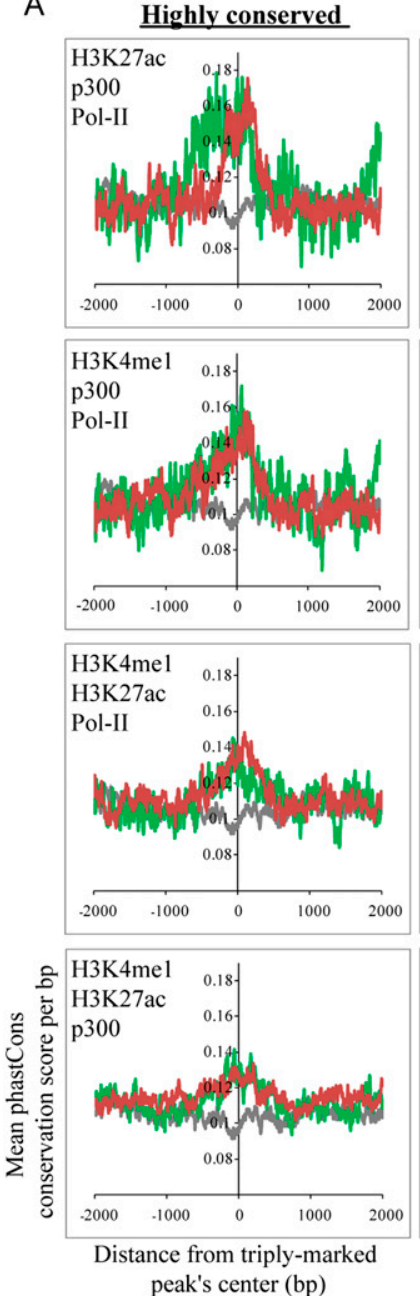

peak's center (bp)

MB-specific triply-marked regions -

MT-specific triply-marked regions -

Random regions
Poorly conserved
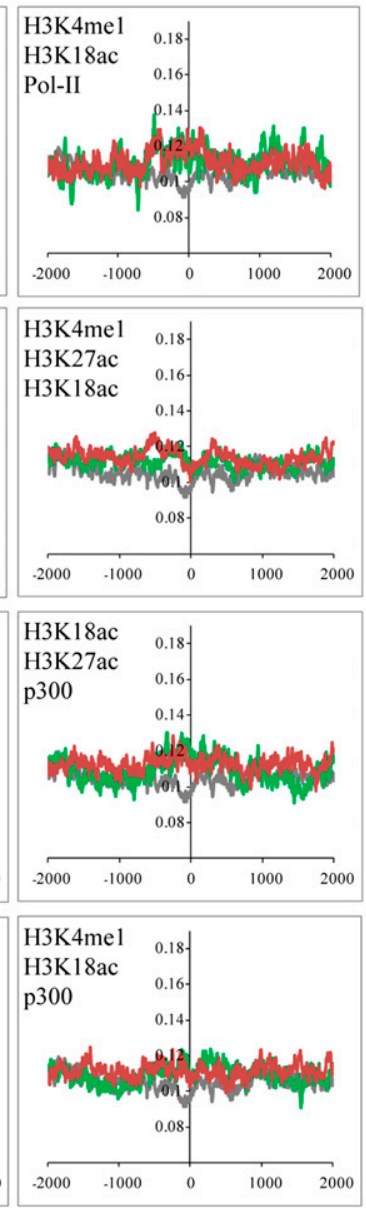

-
-
-
B

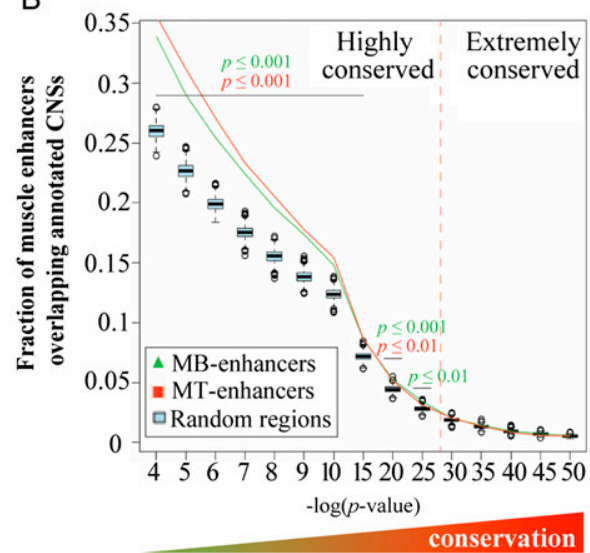

C

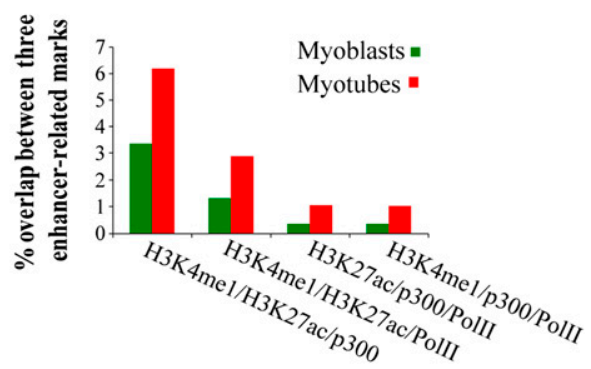

D

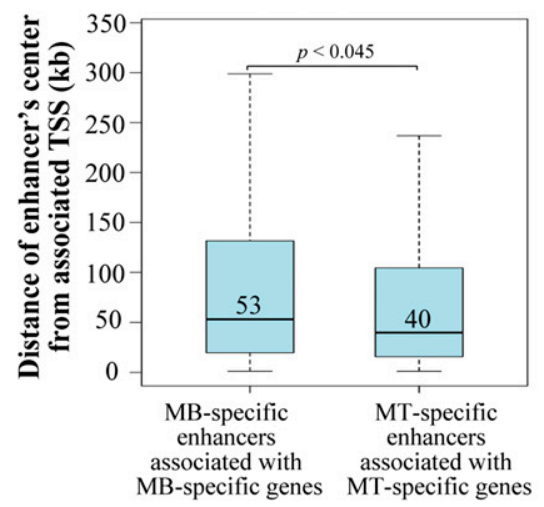

Figure 2. Conservation and condition specificity of muscle enhancers. (A) The average phastCons conservation scores per base pair were plotted $\pm 2 \mathrm{~kb}$ of the center of condition-specific triply marked peaks. The four most highly conserved and four least wellconserved groups are indicated as highly and poorly conserved, respectively. The panel of highly conserved groups refers to the following enriched combinations of marks: H3K4me1/H3K27ac/p300 (3442 and 5054), H3K4me1/H3K27ac/Pol II (1213 and 2219), H3K4me1/p300/Pol II (477 and 1051), and H3K27ac/p300/Pol II (333 and 932), where the indicated numbers of genomic fragments are shown parenthetically for myoblasts and myotubes, respectively. (B) Enrichment of myoblast and myotube enhancers at CNSs. A database of noncoding genomic human-rodent conserved sequences was intersected with myoblast or myotube enhancers or 1000 random sets of sequences of similar length and composition. The fractions of myoblast and myotube enhancers (green and red lines, respectively) and the respective distributions of control random sequences (cyan box plots) overlapping annotated CNSs are presented as a ranked series (shown from left to right) based on the increasing degree of CNS sequence conservation (where the smallest $P$-values indicate the most extreme degree of conservation). Muscle enhancers are associated with CNSs that are highly conserved (left end of the graph), but statistical significance is lost when they are compared with sets of extremely conserved CNSs (far right on the graph). (C) Coordinated accumulation of multiple marks at enhancers is higher in the differentiated state. Quantitation of triply marked enhancerrelated peaks of the four most highly conserved groups. $(D)$ Distributions of the median distance from the center of condition-specific enhancers to the TSS of their nearest associated condition-specific gene (Student's two-sample $t$-test).

(Fig. 2C), suggesting that the concerted accumulation of multiple marks at enhancers is more tightly associated with the differentiated state. Indeed, most of the enhancers we detected were myotube-specific $(53 \%)$, whereas a smaller fraction appeared to be marked exclusively in myoblasts $(31 \%)$ or constitutively throughout differentiation $(16 \%)$. The median distance of enhancers to the transcription start sites (TSSs) of their nearest associated 
known genes was $\sim 50 \mathrm{~kb}$ (Supplemental Fig. S1E), consistent with previous reports (Kim et al. 2010; Rada-Iglesias et al. 2011). Since active, condition-specific enhancers are likely to amplify expression of associated genes, we linked enhancers with their nearest condition-specific genes (expressed $\geq 1.5$-fold higher in one condition vs. the other) and discovered that the median enhancer-promoter distance for myotubes was significantly shorter $(P<0.045)$ than the corresponding distance in myoblasts (median distances were $39.7 \mathrm{~kb}$ vs. $53 \mathrm{~kb}$ for myotube and myoblast enhancers, respectively) (Fig. 2D). Since nearly twice as many condition-specific enhancers were identified in myotubes as in myoblasts, we performed a series of random sampling analyses that confirmed our original observation and ruled out the possibility that our observation was affected by the unequal number of enhancers analyzed (Supplemental Fig. S1F). This observation indicates that a reduction in the distances between active enhancers and their active linked promoters may be a feature of muscle differentiation.

\section{Enhancers are associated with spatially constrained chromatin marks and gene expression}

We next generated chromatin state maps for conditionspecific and constitutive enhancers, plotting H3K4mel, H3K27ac, p300, and Pol II enrichment, together with several other histone acetylation and methylation marks that we investigated previously (Fig. 3A; Supplemental Fig. S2A; Asp et al. 2011). We found that the four most conserved enhancer-related features (H3K4me1, H3K27ac, p300, and Pol II) had overlapping patterns, concentrating within $\pm 0.5 \mathrm{~kb}$ of the center of our defined enhancers. $\mathrm{H} 4 \mathrm{~K} 12 \mathrm{ac}, \mathrm{H} 3 \mathrm{~K} 9 \mathrm{ac}$, and H3K18ac were noticeably less focused within this region, and the latter mark contrasts strongly with $\mathrm{H} 3 \mathrm{~K} 27 \mathrm{ac}$, although both modifications are catalyzed by p300 (Jin et al. 2011).

To determine whether these enhancer signatures are specific for muscle, we examined existing ChIP-seq data for enhancer-associated marks in several other mouse tissues (Visel et al. 2009; De Santa et al. 2010; Kim et al. 2010; Mikkelsen et al. 2010). In these tissues, we identified relatively fewer marks or binding events corresponding with our identified muscle enhancers, and in those cases, their binding pattern was considerably more diffuse (Fig. 3B). Interestingly, however, H3K27ac deposition in murine adipocytes and preadipocytes (Mikkelsen et al. 2010) concentrated near the center of muscle enhancers and most strongly resembled the pattern in muscle (Fig. 3B). Gene ontology (GO) analysis of the nearest genes linked with this common set of muscle/adipocyte enhancers pointed to an association with functional categories related to both adipocyte and muscle cell function, including adipocytokine, insulin, and Wnt signaling, as well as muscle development (Supplemental Table S3; Liu et al. 2010; Mikkelsen et al. 2010). Indeed, since adipocytes and skeletal muscle cells are derived from a common precursor (mesenchymal stem cells ([MSCs]) and fat cells share a common gene expression program or "myogenic signature" (Seale et al. 2008), our data suggest that these two related cell types also share a set of enhancers that may be relevant for the expression of these genes. Other studies have shown cell type specificity in the partitioning of H3K4me1 and H3K27ac (Heintzman et al. 2007, 2009), and our data extend these findings to other enhancerassociated marks. Furthermore, our data reinforce the notion that we identified functionally relevant musclespecific regulatory elements.

Next, we clustered our condition-specific enhancers based on histone modifications and, overall, observed similar patterns in both conditions (Fig. 3C). Notably, $40 \%$ of myoblast-specific enhancers and $51 \%$ of myotube-specific enhancers were marked by Pol II. While enhancers with Pol II were almost always marked with H3K4me1 and H3K27ac and generally bound by p300, the majority of enhancers marked with p300 were devoid of Pol II (Fig. 3C).

We merged our ChIP-seq data with genome-wide expression profiles of $\mathrm{C} 2 \mathrm{C} 12$ myoblasts and myotubes (Liu et al. 2010) to determine whether the appearance of enhancer marks correlated with condition-specific changes in gene expression. First, we assigned enhancer peaks to individual genes ("linked genes") $\leq 20 \mathrm{~kb}$ from a known TSS to reduce the frequency of arbitrary annotation (Fig. 4A). When compared with a control group, the set of genes associated with myoblast-specific enhancers was strongly enriched for genes that are more highly expressed in myoblasts as compared with myotubes (Fig. 4A). An even stronger association was found between myotube-specific enhancers and genes that are more highly expressed in myotubes. We next clustered linked genes using GO functional categories (Fig. 4B). Genes associated with myoblast-specific enhancers and constitutive enhancers were primarily associated with cell cycle, cell migration, cytoskeleton, tube development, and regulation of myofibril and sarcomere structure. Myotube-specific enhancers were associated with genes required for muscle differentiation, including muscle and myofibril development, as well as nitrogen metabolism. Indeed, our data suggest that all muscle regulatory factors (MRFs), MyoD1, Myog, Myf5, and Myf6, are under the control of myotube-specific enhancers (Supplemental Fig. S1D; Asp et al. 2011; data not shown). Thus, our data indicate that condition-specific enhancers play a strong inductive role in expression of their associated genes vital to initiation and completion of myogenic differentiation (Supplemental Table S4).

\section{Noncoding transcripts are linked with muscle enhancer activity}

Enhancers are known to recruit Pol II, which results in transcription of short $(<2-\mathrm{kb})$ eRNAs and long ncRNAs (Creyghton et al. 2010; De Santa et al. 2010; Kim et al. 2010). We found a significant association between Pol II enrichment and combinations of enhancer marks (Fig. $3 \mathrm{~A}, \mathrm{C})$. In addition, $\sim 7 \%$ of condition-specific enhancers were trimethylated on $\mathrm{H} 3 \mathrm{~K} 36$ in a condition-specific manner throughout the entire enhancer region, indicative of prominent extragenic transcriptional activity that could be associated with large intervening ncRNAs (lincRNAs) and enhancer activity (Fig. 3A,C; Guttman et al. 2009). 

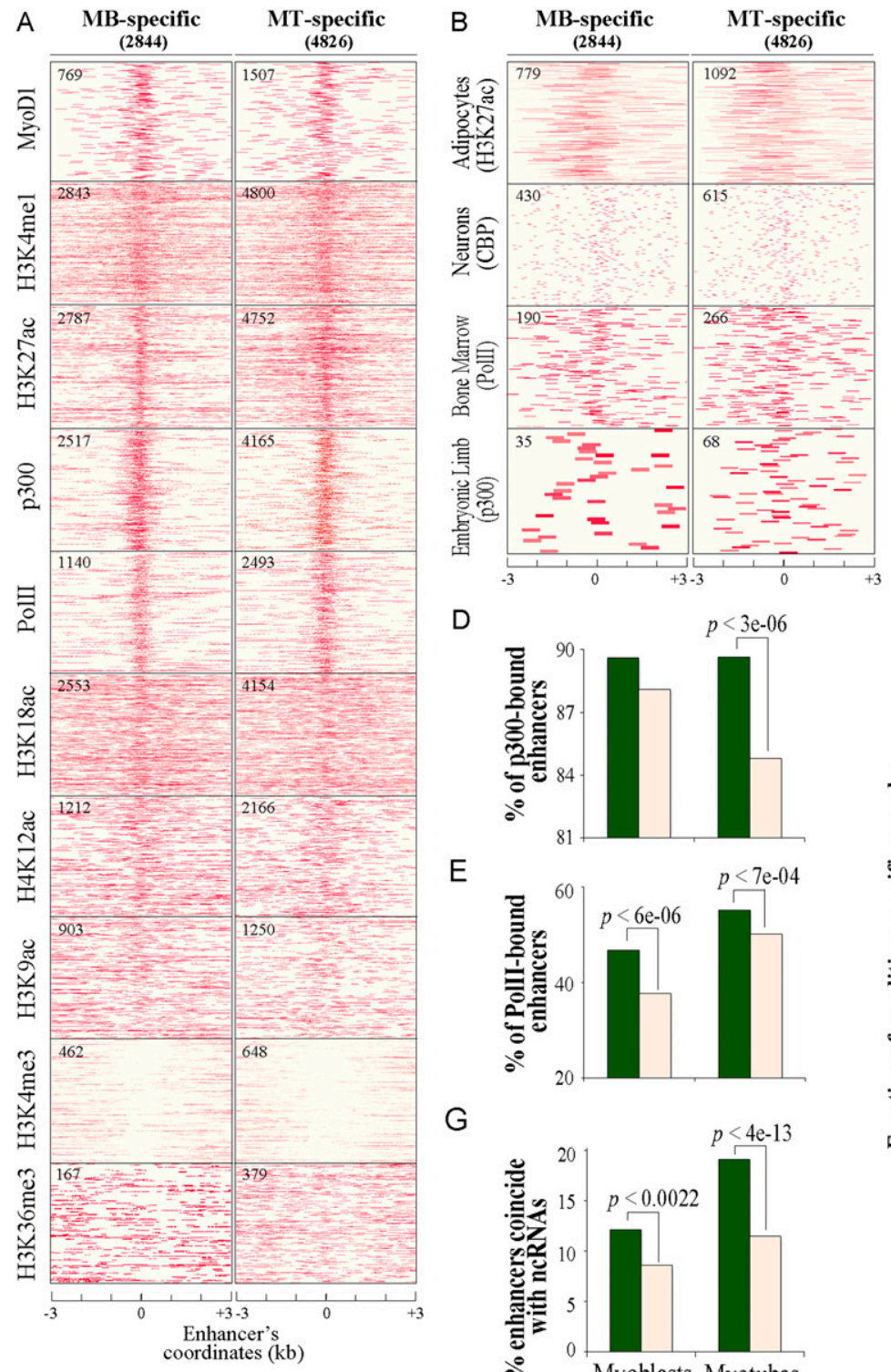

D

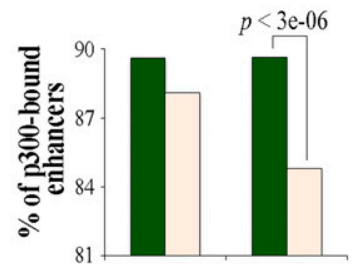

E

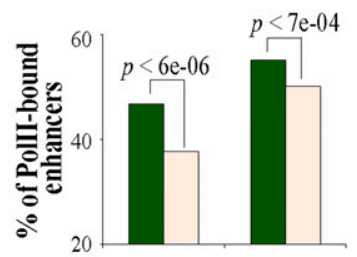

G
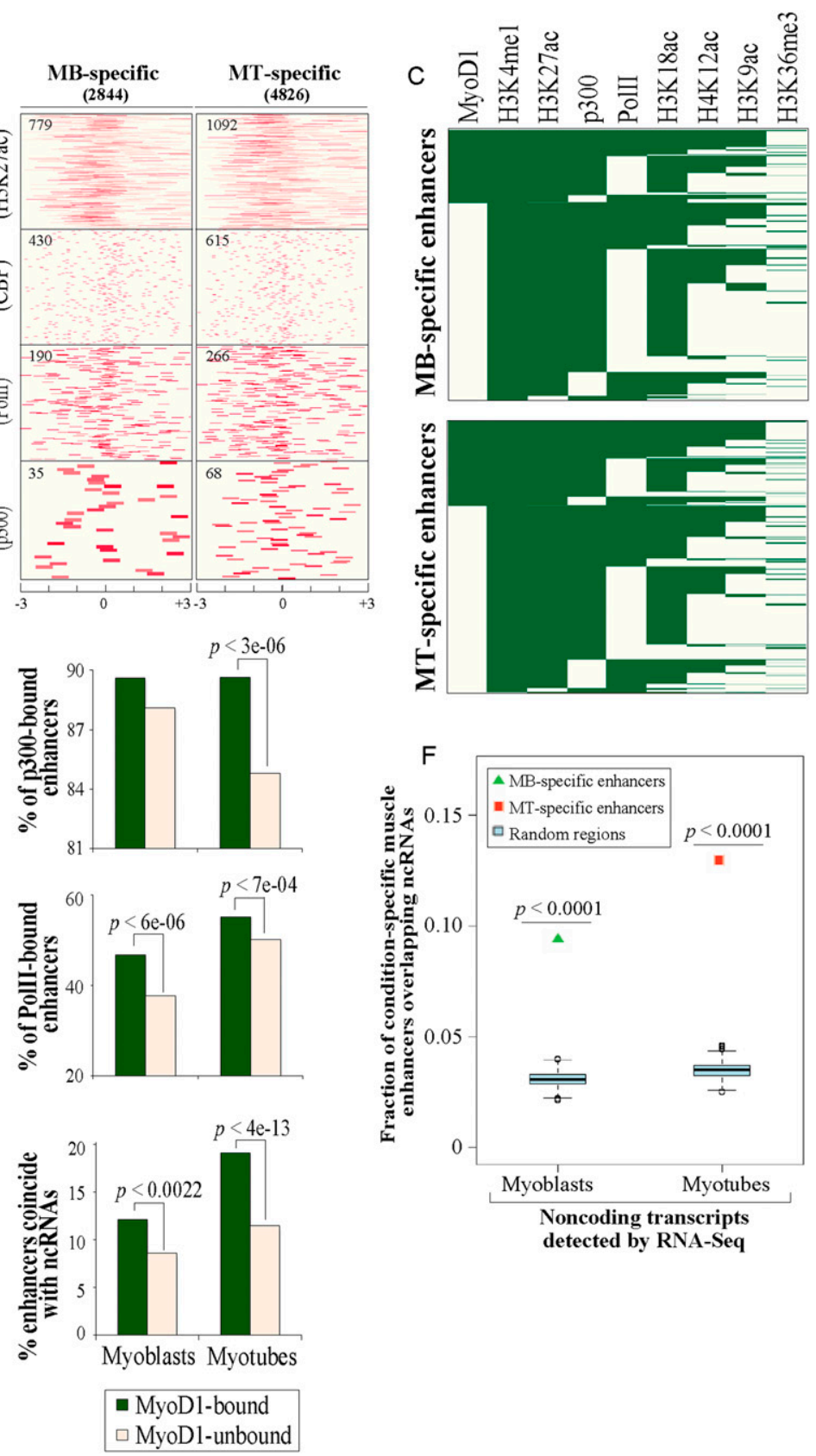

Figure 3. Muscle enhancers are uniquely associated with spatially constrained chromatin marks. (A) Chromatin state maps of enhancer-related markers and several other histone marks within a region $\pm 3 \mathrm{~kb}$ of the center of condition-specific enhancers. Data for MyoD1-binding events were obtained from Cao et al. (2010). p300- and H3K27ac-binding events (enriched tags) were identified using Qeseq (Supplemental Material). ChIP-seq data for p300 and H3K27ac were generated in this study, and all other data were published previously (Asp et al. 2011). The number of condition-specific enhancers significantly enriched with the indicated mark is indicated in the top left corner of each map. $(B)$ Correspondence between muscle enhancers and enhancers in nonmuscle tissues. Enriched binding events of enhancer-related marks obtained from several nonmuscle mouse tissues (Visel et al. 2009; De Santa et al. 2010; Kim et al. 2010; Mikkelsen et al. 2010) corresponding to condition-specific muscle enhancers are plotted as indicated. (C) Clustering of MyoD1, enhancer features, and other histone marks based on their deposition at condition-specific muscle enhancers. $(D, E)$ Recruitment of p300 and Pol II to MyoD1-bound enhancers. Recruitment of p300 (D) and Pol II $(E)$ is significantly higher on enhancers bound by MyoD1 (two-proportion $z$-test). (F) Enrichment of condition-specific enhancers with transcribed ncRNAs deduced from RNA-seq (Trapnell et al. 2010). ncRNAs were overlapped with condition-specific enhancers or random data sets (Supplemental Material). The fractions of condition-specific enhancer and the respective distributions of random, control sequences (cyan box plots) overlapping ncRNAs are presented. $(G)$ Coincidence of ncRNAs with enhancers is significantly higher on MyoD1-bound enhancers. 

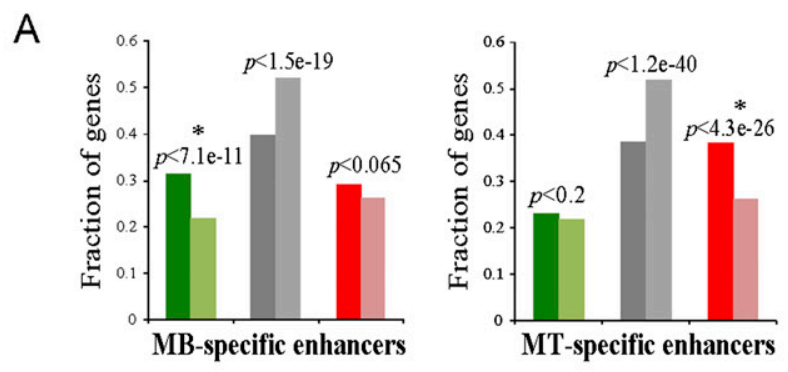

a Genes highly expressed in MB (Obs.)

Genes highly expressed in $\mathrm{MB}$ (Exp.)

a Constitutively expressed genes (Obs.)

- Constitutively expressed genes (Exp.)

- Genes highly expressed in MT (Obs.)

nenes highly expressed in MT (Exp.)
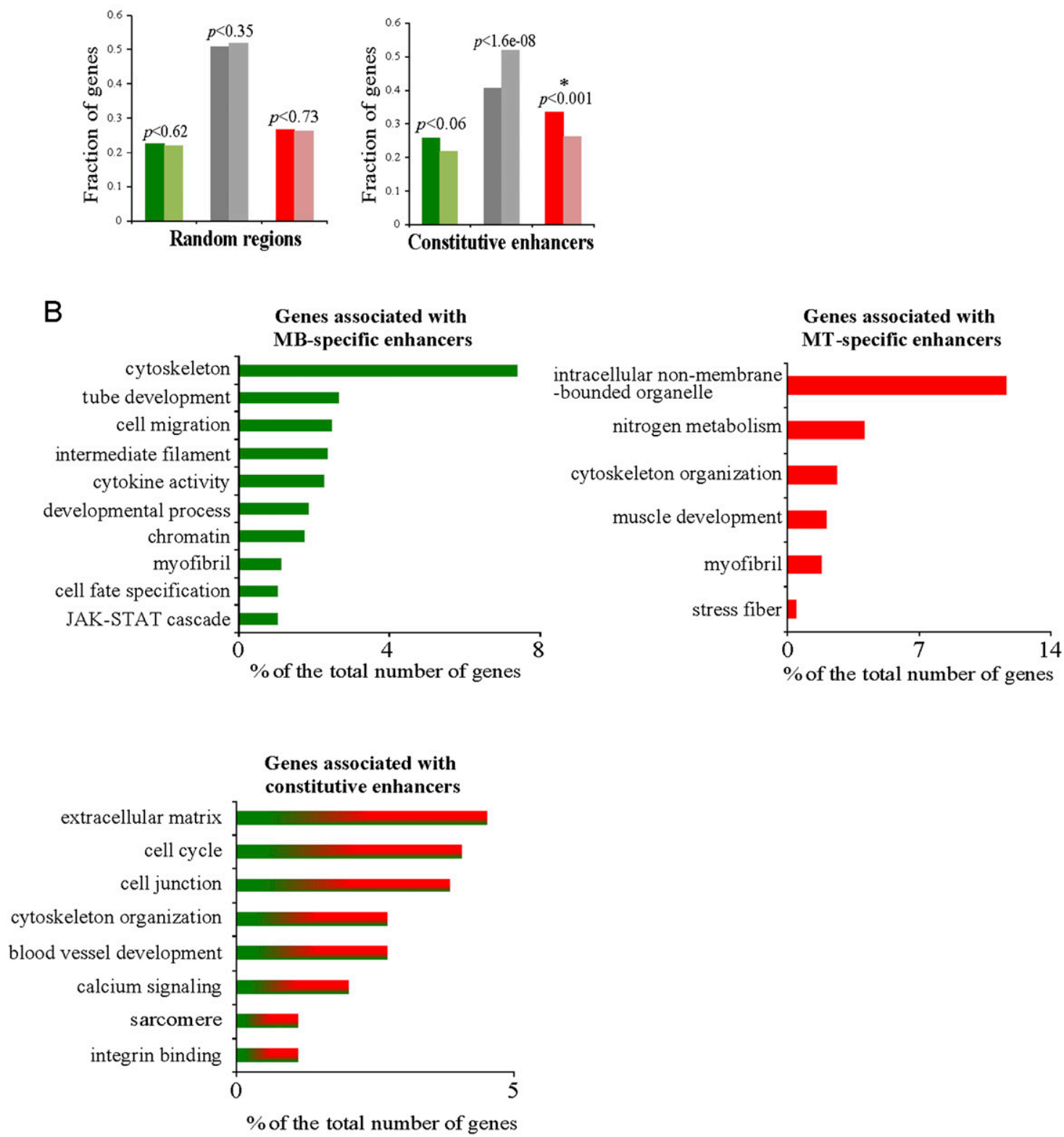

Figure 4. Correlation between assembly of condition-specific enhancers and levels of transcripts of associated protein-coding genes. (A) Condition-specific enhancers (top panel) and constitutive enhancers, along with random genomic regions (bottom panel), are shown. Observed (Obs) and expected (Exp) fractions for each group of genes (highly expressed in myoblasts, highly expressed in myotubes, and constitutively expressed) are shown together with their respective $P$-values, as calculated using a $\chi^{2}$ statistical test. Asterisks indicate statistical significance as compared with expected fractions $(P$-values $<0.05)$. Expected fractions were calculated for each of the four examined data sets on the basis of the relative frequency of each group of associated genes (located at a distance $\leq 20 \mathrm{~kb}$ from a known TSS) with respect to all genes associated with the tested data set. Observed frequencies were determined by quantifying the entire list of genes represented by the microarray based on the three gene categories. $(B)$ GO categories for genes associated (located at a distance $\leq 20 \mathrm{~kb}$ from a known TSS) with condition-specific and constitutive enhancers. GO categories with $P$-values $<0.05$ are shown. 
To further investigate whether muscle enhancers coincide with ncRNA, we merged our enhancer compendium with transcriptome profiling (RNA-seq) data for C2C12 cells before and after differentiation (Trapnell et al. 2010) and filtered out transcripts with coding potential (Supplemental Material). Association of condition-specific enhancers with myoblast and myotube ncRNAs was statistically significant and more than twofold enriched compared with random genomic sets (Fig. 3F; Supplemental Table S5). Strikingly, these results indicated that $\sim 10 \%$ of condition-specific muscle enhancers are characterized by sites of active, noncoding transcription, consistent with a previous report linking enhancers with overlapping ncRNAs in a variety of cell types (Fig. 3F; Supplemental Table S5; Cabili et al. 2011). Importantly, we confirmed the expression of several ncRNAs that overlapped with our condition-specific enhancers (Fig. 5F). Approximately 60\% of condition-specific enhancers that overlapped with transcribed regions (based on RNA-seq) displayed significant levels of Pol II recruitment. Notably, genes associated with these transcriptionally active myoblast enhancer regions were enriched for GO categories related to muscle formation (including positive regulation of growth and myofibril and skeletal muscle development clusters) (data not shown). Several key regulators, such as Msx1, Pax7, Fgfrl1, and Six1, which are transcribed in myoblasts (Liu et al. 2010), were among these genes. Similarly, genes associated with transcriptionally active myotube enhancers such as Tnnt2, Myod1, Neb, and Myog are linked to musclerelated functions, including sarcomere organization, muscle cell differentiation, and cell morphogenesis. Although we cannot rule out the possibility of limited coding potential of some of the ncRNAs analyzed here, our studies indicate that we identified a substantial group of muscle enhancers associated with noncoding transcripts.

\section{Enrichment of TFs on muscle enhancers}

One prediction is that active muscle enhancers should be enriched for TF-binding sites (TFBSs) associated with factors required for the execution of muscle differentiation programs, such as MyoD1 and Mef2c, since it is known that co-occupancy by multiple TFs can assist in the assembly of active tissue-specific enhancers. We used the CLOVER algorithm (Frith et al. 2004) to examine which TFBS (represented by position weight matrices [PWMs]) are enriched in our collection of condition-specific enhancers (Supplemental Material; Supplemental Table S6). We found that the majority of TF motifs were significantly clustered within a $1-\mathrm{kb}$ region centered around the middle of the enhancers, supporting the notion that enhancers could be assembled within constrained genomic regions bound by specific sets of TFs. MyoD1 and related E-box-binding motifs were significantly enriched within this restricted 1-kb window (Supplemental Table S6). Indeed, $46 \%$ of myoblast-specific and $80 \%$ of myotube-specific enhancers exhibited enrichment for MyoD1 PWMs within this window. In addition, a consensus MEF2A-binding motif was enriched in $\sim 27 \%$ of myoblast- and myotubespecific enhancers. An alternative MEF2A-binding motif recently identified in cardiomyocytes (He et al. 2011) was exclusively enriched in myotube-specific enhancers (Supplemental Fig. S2B; Supplemental Table S6).

Our analyses further implicated binding by several TFs with roles in myogenesis. For example, myoblast-specific enhancers were exclusively enriched with $\mathrm{Pbx} 1$ and $\mathrm{p} 53$ (Supplemental Fig. S2B; Supplemental Table S6). Myotube-specific enhancers were exclusively enriched with PPAR $\gamma$ and c-Myb (Supplemental Fig. S2B; Supplemental Table S6). Other TFs previously implicated in the regulation of myogenesis, such as RP58, Runx, and Jdp2, were predicted to bind muscle enhancers in both conditions. Our results suggest that these TFs could execute their function through direct binding to muscle enhancers.

\section{MyoD1 recruitment to muscle enhancers}

MyoD1 is known to play a key role in myogenesis, and genome-wide studies have revealed binding events in promoters as well as intergenic regions in myoblasts and myotubes (Blais et al. 2005; Cao et al. 2010). MyoD1 binding is generally associated with gene activation (Cao et al. 2006). We therefore took advantage of experimentally determined MyoD1-binding sites (23,271 and 25,956 in $\mathrm{C} 2 \mathrm{C} 12$ myoblasts and myotubes, respectively) established through genome-wide ChIP-seq analysis (Cao et al. 2010 ) and found that $\sim 20 \%$ of our predicted MyoD1binding sites were occupied by MyoD1. This finding suggests that additional sequence-specific factors and/or the chromatin environment, dictated by histone modifications described here (and possibly others), play an important role in MyoD1 recruitment at enhancers.

To explore a role for MyoD1 at enhancers, we used these previously published MyoD1 ChIP-seq data and confirmed condition-specific binding to a subset of our muscle enhancers (Supplemental Fig. S2C). To determine whether genome-wide correlations exist between MyoD1 binding, a given set of chromatin modifications, factor recruitment, and acquisition of an active enhancer state, we merged the experimentally determined MyoD1-binding events with our data (Fig. 3C). We found that MyoD1 was recruited to $27 \%$ of myoblast-specific and $31 \%$ of myotube-specific enhancers (Fig. 3C), and, remarkably, the binding of this factor was concentrated very close to the center of these enhancers (Fig. 3A). Among 855 myoblast-specific enhancers that were bound by MyoD1 in either condition, the majority was bound exclusively in myoblasts $(31 \%)$ or in both myoblasts and myotubes (60\%) (Supplemental Fig. S2D). Thus, in myoblasts, the presence of MyoD1 was highly correlated with assembly of enhancers. Similarly, MyoD1 binding was also strongly correlated with assembly of myotube enhancers, since the majority of enhancers bound this TF in either myotubes specifically $(28 \%)$ or both myoblasts and myotubes (57\%) (Supplemental Fig. S2D). Given the striking overlap between MyoD1 and muscle enhancers, we focused the remainder of our studies on MyoD1-mediated regulation of these enhancers.

Genes linked to upstream MyoD1-bound enhancers included Mef2c, MyoD1, Myl2, Myog, Msx1, and other key regulators of skeletal muscle development and sarcomere 
formation (Supplemental Fig. S2D). Interestingly, 15\% of myotube-specific enhancers were bound by MyoD1 exclusively in myoblasts, suggesting that assembly of a specific set of myotube enhancers might be suppressed by MyoD1 prior to differentiation and that enhancers could be activated during differentiation upon MyoD1 removal. Alternatively, MyoD1 may be required to be present in myoblasts to "prime" these enhancers for complex assembly during the initial stages of differentiation (Supplemental Fig. S2D).

We found that $\sim 90 \%$ of condition-specific enhancers bound by MyoD1 were co-occupied with p300, in agreement with the known biochemical interactions between p300 and MyoD1 (Fig. 3C,D; Yuan et al. 1996). Among myotube-specific (but not myoblast-specific) enhancers, the fraction of MyoD1-bound enhancers co-occupied by p300 was significantly higher $\left(P<3 \times 10^{-6}\right.$, by twoproportion $z$-test) than the fraction of enhancers without MyoD1 that were bound by p300 (Fig. 3D), suggesting that MyoD1 might play a more important role in p300 recruitment during differentiation. In addition, and consistent with a role for MyoD1 in promoting Pol II engagement at promoters of skeletal muscle genes /Cao et al. 2006), we found that $47 \%$ of myoblast-specific and $55 \%$ of myotube-specific enhancers that were bound by MyoD1 were co-occupied by Pol II (Fig. 3E). MyoD1 binding significantly increased Pol II recruitment at enhancers (by $24 \%$ and $9 \%$ in myoblasts and myotubes, respectively) as compared with enhancers that were not bound by MyoD1 (Fig. 3E). However, overall, the majority of Pol IIbound enhancers was not bound by MyoD1, suggesting that recruitment of Pol II to muscle enhancers may be largely mediated through MyoD1-independent mechanisms. Taken together, these analyses point toward a compelling association between MyoD1 binding and recruitment of p300 and Pol II to muscle enhancers and suggest an important and widespread role for this MRF in promoting deposition of these factors.

\section{A functional role for MyoD1 recruitment to enhancers}

In the embryo, MyoD1 is expressed in myoblasts, which are committed to the myogenic lineage (Sassoon et al. 1989). Muscle development is not abolished in mice lacking MyoD1, likely due to functional compensation by another MRF, Myf5, which is strongly up-regulated in null animals (Rudnicki et al. 1992). Nevertheless, MyoD1 knockout $\left(\mathrm{MyoD}^{-/-}\right)$animals exhibit marked differentiation defects, including significant delays in the expression of muscle-specific genes, such as myogenin (Megeney et al. 1996). Therefore, we investigated the effects of MyoD1 ablation on the assembly of muscle enhancers. We isolated primary myoblasts from wild-type and $M y o D 1^{-/-}$mice (Rudnicki et al. 1992) and confirmed that MyoD1 was depleted from a subset of enhancers in null myoblasts (Supplemental Fig. S2E). Next, we assessed the impact of MyoD1 depletion on the acquisition of enhancer signatures by examining deposition of H3K4me1, H3K27ac, Pol II, and p300 (Fig. 5A-D) at selected myoblast-specific enhancers bound by MyoD1. Interestingly, we detected striking reductions in each of these marks at the MyoD1- bound enhancers. In sharp contrast, enrichment was not altered at any enhancers that were not bound by MyoD1. These controls indicate that diminution of each histone mark and recruitment of Pol II and p300 was a direct result of MyoD1 ablation and strongly suggest that MyoD1 plays a prominent role in the acquisition of an enhancer state (Fig. 5A-D).

Furthermore, we analyzed expression of a group of protein-coding genes closest to myoblast-specific enhancers that recruited MyoD1. Importantly, we found that expression of several associated genes was significantly reduced in the absence of MyoD1 as compared with wildtype myoblasts (Fig. 5E). Furthermore, we discovered that MyoD1 binding significantly increased the frequency with which condition-specific enhancers coincided with ncRNA transcripts (by $41 \%$ and $67 \%$ in myoblasts and myotubes) as compared with enhancers that were not bound by MyoD1 (Fig. 3G). We therefore examined the impact of MyoD1 loss on the transcription of ncRNAs that coincided with muscle enhancer regions. To this end, we used RT-PCR to measure expression of several ncRNAs in wild-type and $M y o D 1^{-/-}$primary myoblasts (Fig. 5F). Interestingly, we found that in the majority of cases, expression of these ncRNAs was significantly altered in cells lacking MyoD1. In contrast, we found that the expression of ncRNAs transcribed from enhancers that did not bind MyoD1 was not affected in $M y o D 1^{-/-}$ myoblasts (Fig. 5F). We conclude that MyoD1 acts (1) locally to direct expression of ncRNAs at enhancers and (2) at a distance to drive expression of linked, downstream protein-coding genes.

\section{MyoD1 is mechanistically linked to recruitment of Set7, an H3K4 monomethylase}

Next, we sought to elucidate the mechanisms responsible for MyoD1-dependent assembly of enhancers. First, given the accumulation of $\mathrm{H} 3 \mathrm{~K} 4 \mathrm{me} 1$ at enhancers, we examined the recruitment of Set7/Set9, an enzyme that catalyzes H3K4 monomethylation. We used qChIP to examine the recruitment of this enzyme to a subset of our enhancers and determined whether the enzyme was recruited in a MyoD1-dependent manner. We found that Set7 was recruited to a cohort of enhancers (Fig. 5G). Interestingly, in MyoD $1^{-/-}$myoblasts, the recruitment of Set7 was significantly diminished at each MyoD1-bound enhancer but not at enhancers without MyoD1, indicating that recruitment of Set7 to this group of enhancers depends on MyoD1. These results are consistent with a recent study demonstrating direct MyoD1/Set7 interactions on the MCK enhancer and the importance of Set 7 for promoting myoblast differentiation via regulation of $\mathrm{H} 3 \mathrm{~K} 4 \mathrm{mel}$ deposition (Tao et al. 2011). Moreover, we detected MyoD1-independent recruitment of Set7 to enhancers, suggesting that this methyltransferase may be recruited by additional TFs.

\section{MyoD1 recruits a cohort of TFs to assemble enhancers in muscle}

To further investigate MyoD1-bound enhancers, we sought to identify binding motifs that are uniquely and specifi- 
A

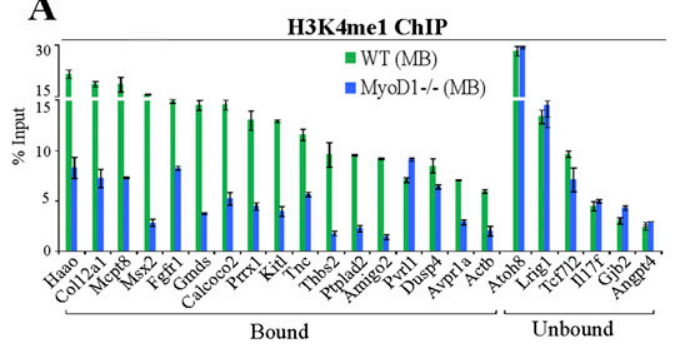

B

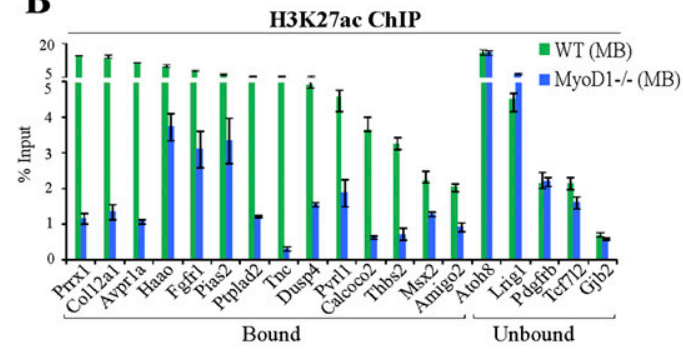

C

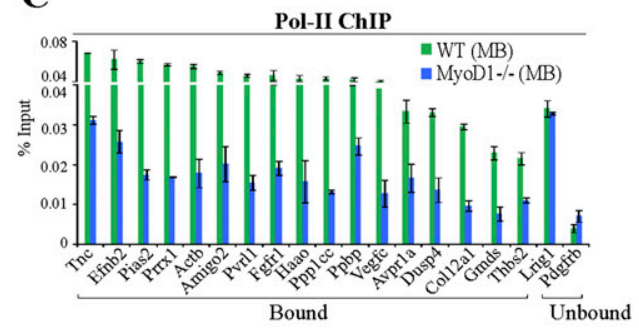

D

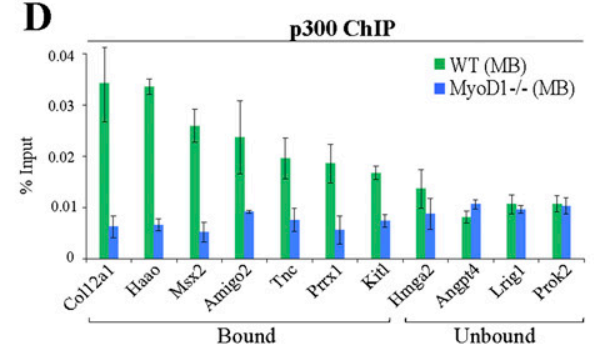

E

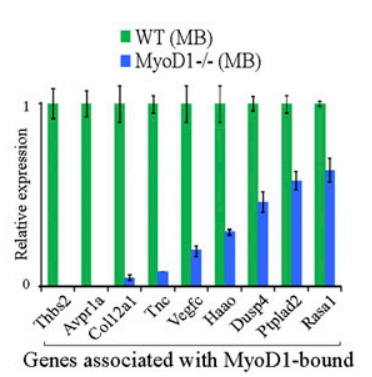

Genes associated with MyoD1-bound
F
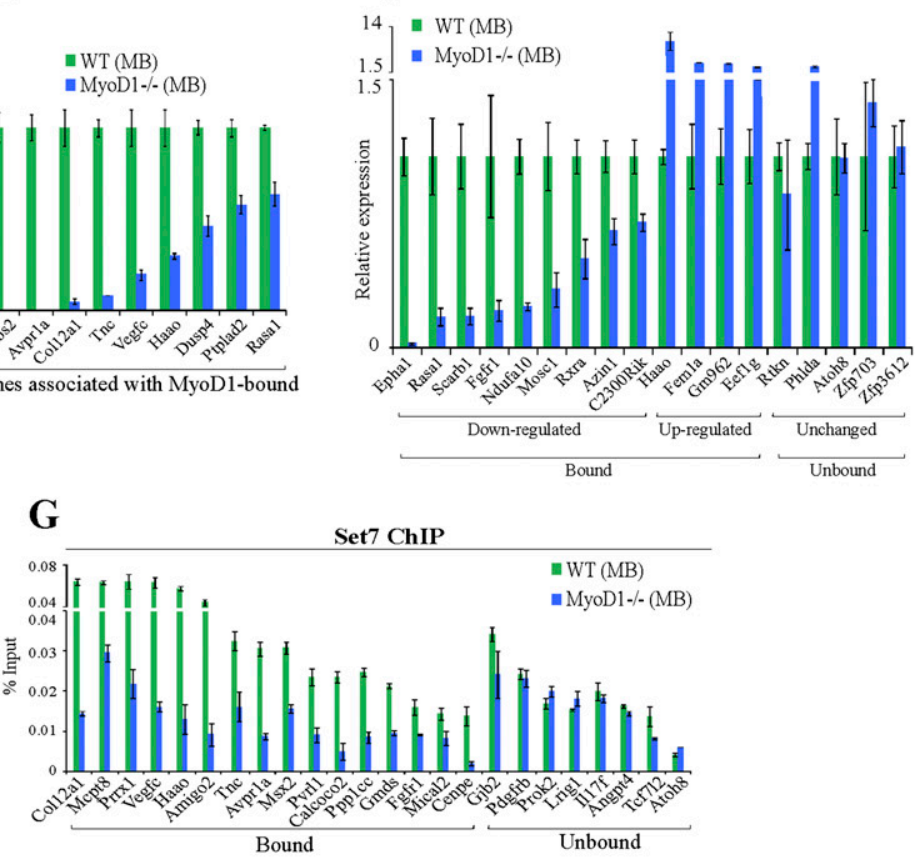

H
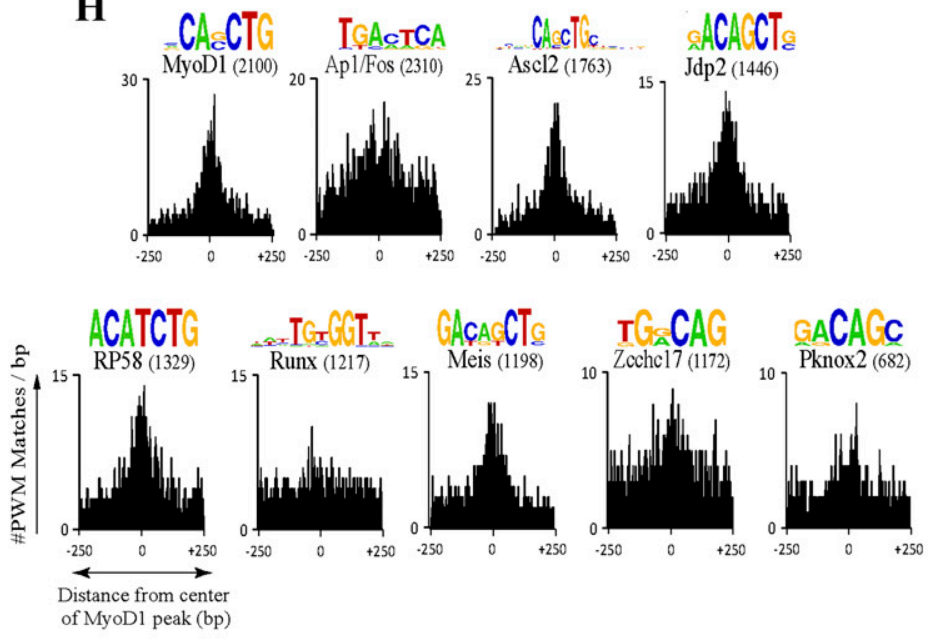

Figure 5. MyoD1 loss results in reduced assembly of MyoD1-bound enhancers. $(A-D)$ qChIP was performed to detect enhancer-related marks-H3K4me1 $(A), \mathrm{H} 3 \mathrm{~K} 27 \mathrm{ac}(B)$, Pol II $(C)$, and p300 $(D)$, as indicated—on MyoD1-bound myoblast-specific enhancers in primary $M y o D 1^{-/-}$and wild-type myoblasts. Several myoblast-specific enhancers not bound by MyoD1 were tested as negative controls and showed insignificant alterations in the levels of all marks tested. (E) RT-qPCR showing the effect of MyoD1 loss on a cohort of genes that are associated with MyoD1-bound, myoblast-specific enhancers. Data from wild-type and MyoD1 $1^{-1-}$ primary myoblasts are indicated. Relative expression of each gene is plotted with respect to primary wild-type myoblasts. ( $F)$ MyoD1 depletion significantly alters expression of ncRNAs transcribed across myoblast-specific enhancers. RNA was obtained from wild-type and $M y o D 1^{-1-}$ primary myoblasts. Expression levels of ncRNAs coinciding with MyoD1-bound enhancers were measured by qPCR. As a control, we measured the expression levels of several other ncRNAs that coincide with enhancers that are not bound by MyoD1. (G) qChIP comparing Set7 recruitment with MyoD1-bound enhancers and enhancers that do not bind MyoD1. $(H)$ Distributions of PWMs enriched in MyoD1bound myoblast-specific enhancers. For each predicted binding motif, the accumulative number of matches per 1 bp was plotted across the region ( $\pm 250 \mathrm{bp}$ ) surrounding the center of observed MyoD1-binding sites (Cao et al. 2010). Error bars for all qChIP and RT-qPCR data represent the standard error of the mean (SEM).

cally overrepresented at these enhancers. We searched for TF-binding motifs enriched within a restricted window $( \pm 250 \mathrm{bp}$ ) around the center of MyoD1-binding sites. Our analysis demonstrated that the predicted distributions of several TFs, including Jdp2, Meis, c-Jun, Ascl2, RP58, and Runx, strikingly paralleled the experimentally identified MyoD1-binding sites in myoblasts (Fig. 5H; Supplemental Material), although they were more broadly distributed 
than MyoD1 within this window. This analysis prompted us to examine whether MyoD1-bound enhancers are indeed populated by these TFs. We initially selected a set of 20 myoblast-specific enhancers bound by MyoD1 that were predicted to recruit Jdp2, Meis, c-Jun, and Runx, since these factors play a role in myogenesis, and performed qChIP experiments in C2C12 myoblasts and myotubes. As controls, we also included myotube-specific enhancers that were not enriched for the predicted signatures and genomic regions upstream of genes that were never expressed in muscle. Interestingly, we showed that each of these factors was bound specifically in the vicinity of their predicted binding sites in a condition-specific manner (Fig. 6A-D). In contrast, controls lacking these predicted TFBSs showed no enrichment for any factor. Furthermore, qChIP analysis using antibodies against a factor that was not predicted to bind these enhancers (E2F4) confirmed the specificity of these binding events (Fig. 6A-D). These findings indicate that our in silico approach, coupled with genomewide ChIP-seq data, was able to accurately predict the binding of several TFs to enhancers in a conditionspecific manner.

The proximity of each of these factors to MyoD1binding sites suggested that they could be recruited cooperatively to enhancers. To test such a dependence on MyoDl binding, we performed qChIP studies in primary wild-type and $M y o D 1^{-/-}$myoblasts. Interestingly, we found that binding of c-Jun, Jdp2, and Runx was significantly reduced in the absence of MyoD1 (Fig. 6E-G), indicating that all three TFs bind to myoblast enhancers in a MyoD1-dependent manner. Thus, MyoD1 could play a pivotal role in enhancer assembly by recruiting other key TFs with established roles in muscle growth and differentiation. Given the frequency with which these sites occur in our muscle enhancers, we propose that combinations of these TFBSs could constitute new signatures for skeletal muscle enhancers.

To test this assertion in a genome-wide manner, we performed ChIP-seq to identify c-Jun-binding sites in growing myoblasts, since c-Jun has been shown to physically interact with MyoD1 in vivo (Bengal et al. 1992). We found that c-Jun was preferentially enriched in intergenic regions, but, most importantly, $>37 \%$ of our myoblast-specific enhancers had at least one binding site for this factor. Of note, c-Jun was enriched in a striking pattern near the center of these myoblast-specific enhancers (Fig. 7A), and MyoD1 binding was linked to increased, localized enrichment of c-Jun at enhancers (Fig. 7A,B). To examine whether c-Jun participates in the assembly of myoblast enhancers, we suppressed its expression by transfecting $\mathrm{C} 2 \mathrm{C} 12$ myoblasts with an siRNA pool targeting c-Jun (Fig. 7C) and assessed the levels of $\mathrm{H} 3 \mathrm{~K} 4 \mathrm{mel}$ and $\mathrm{H} 3 \mathrm{~K} 27 \mathrm{ac}$ at selected myoblast-specific enhancers bound by c-Jun (Fig. 7D,E). Notably, we detected significant reductions in the levels of these two enhancerrelated histone marks at the c-Jun-bound enhancers but not at selected enhancers that were not bound by c-Jun (Fig. 7D,E). Thus, our studies clearly show that c-Jun participates in the assembly of enhancers in skeletal myoblasts.

\section{MyoD1 dictates an enhancer signature}

The extensive overlap between MyoD1 and muscle enhancers suggested a critical role for this factor in the assembly of enhancers, and our studies in primary $M y o D 1^{-/-}$ myoblasts (Fig. 6) and myotubes (data not shown) provided functional support for this conclusion. To more directly test this prediction and rule out secondary consequences of MyoD1 loss, we performed rescue studies wherein we assessed the impact of MyoD1 re-expression on enhancer assembly in a MyoD1-null background. Here, we stably expressed MyoD1 in primary MyoD $1^{-/-}$ myoblasts (Gerber et al. 1997), and cells harboring the empty vector served as a control. Western blot analysis of nuclear extracts from the resulting cells indicated restoration of MyoD1 at levels approximating those of wildtype cells (Fig. 8A). Next, we examined expression of myogenin, a MyoD1 target gene, as myoblasts reached confluence and initiated differentiation. Myogenin levels were nearly undetectable in $M y o D 1^{-/-}$myoblasts, owing to a delay in differentiation, but were restored in cells reconstituted with MyoD1 (Fig. 8B). Furthermore, null myoblasts complemented with MyoD1 proliferated and underwent differentiation in a manner indistinguishable from wild-type cells, producing myotubes that phenotypically resembled their wild-type counterparts (data not shown). We also examined expression of several genes associated with enhancers bound by MyoD1 (Fig. 5E) and found that expression of MyoD1 significantly augmented their expression, albeit to varying degrees (Fig. 8C; data not shown). Each of these criteria suggests that $M y o D 1^{-/-}$ cells were fully rescued through expression of MyoD1.

Next, we performed qChIP to examine the effect of MyoD1 restoration on deposition of $\mathrm{H} 3 \mathrm{~K} 4 \mathrm{me} 1, \mathrm{H} 3 \mathrm{~K} 27 \mathrm{ac}$, and Pol II at myoblast-specific enhancers. Remarkably, we observed significant increases in $\mathrm{H} 3 \mathrm{~K} 4 \mathrm{me} 1$ deposition and Pol II recruitment in rescued cells (Fig. 8D,E). Strikingly, however, the levels of acetylated H3K27 were not significantly altered (Fig. 8F). Thus, MyoD1 re-expression restored some, but not all, hallmarks of our muscle enhancers. To further explore this finding in myotubes, we differentiated these primary myoblasts and performed qChIP to detect H3K4me1 and H3K27ac at myotubespecific enhancers. Here, we found that deposition of both chromatin marks was significantly elevated, indicating that restoration of MyoD1 expression promoted the reassembly of myotube enhancers (Fig. 8G-I). It is interesting to note that while $\mathrm{H} 3 \mathrm{~K} 27 \mathrm{ac}$ was markedly increased at myotube-specific enhancers, the level of this mark was not augmented at myoblast-specific enhancers. These results suggest that MyoD1 expression may be required in precursor cells (which ultimately give rise to myoblasts) to subsequently allow the correct assembly of myoblast enhancers, since germline deletion of MyoD1 in these precursor cells would abrogate recruitment of p300 and therefore reduce the acquisition of $\mathrm{H} 3 \mathrm{~K} 27 \mathrm{ac}$. In this setting, rescue of MyoD1 expression in myoblasts might 
A

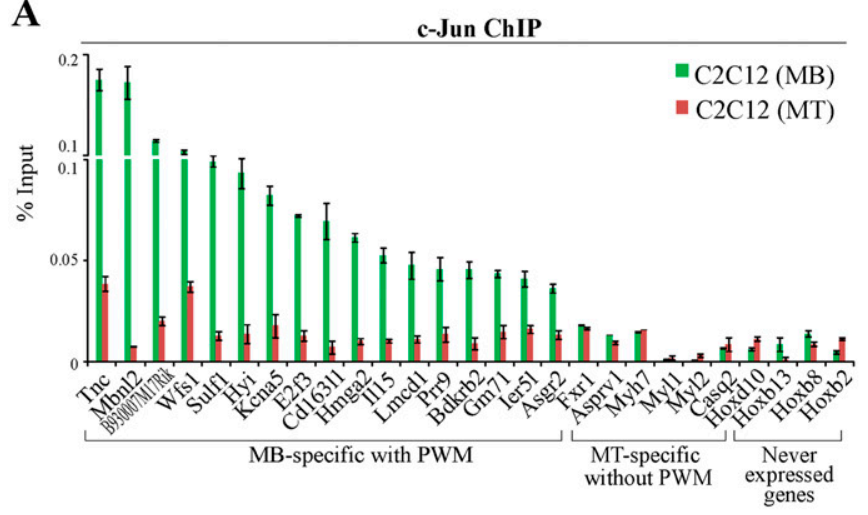

B

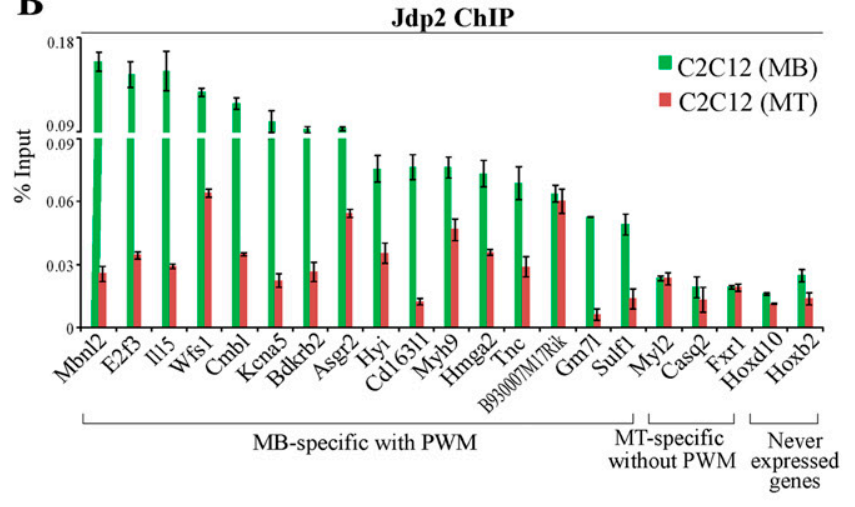

D

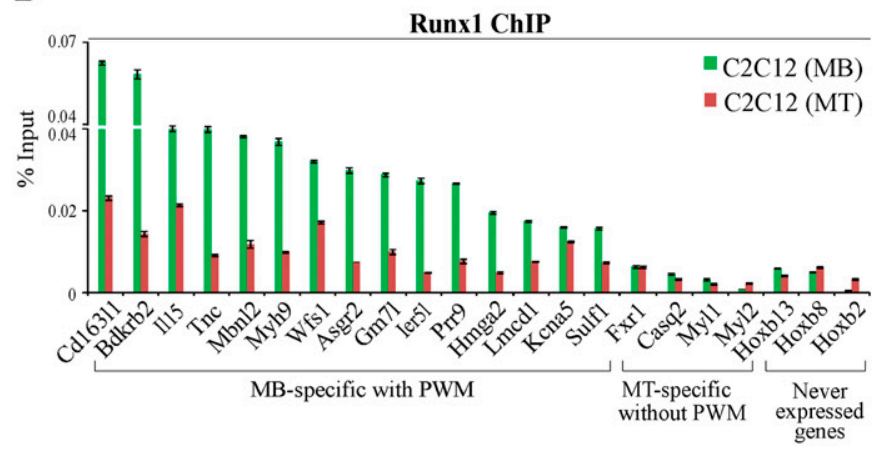

$\mathbf{F}$

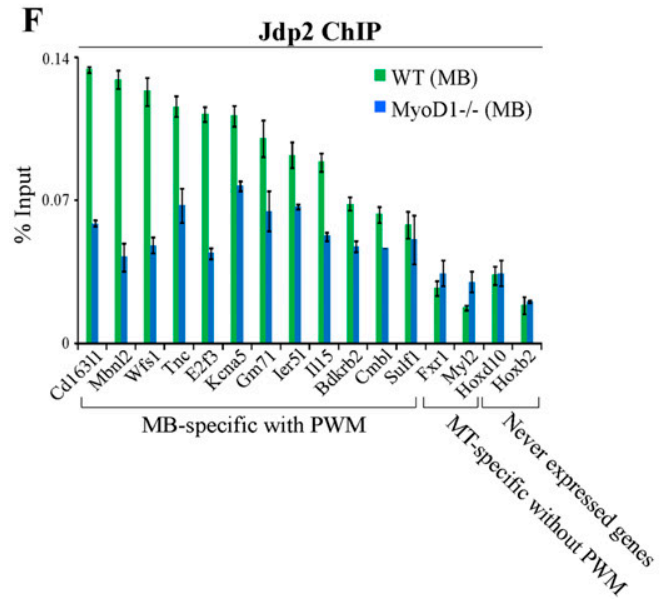

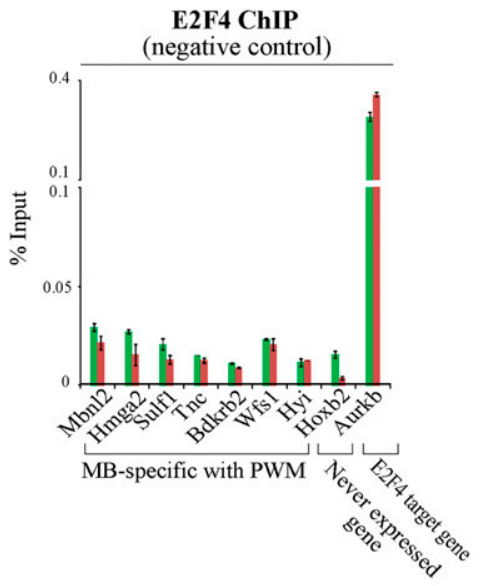

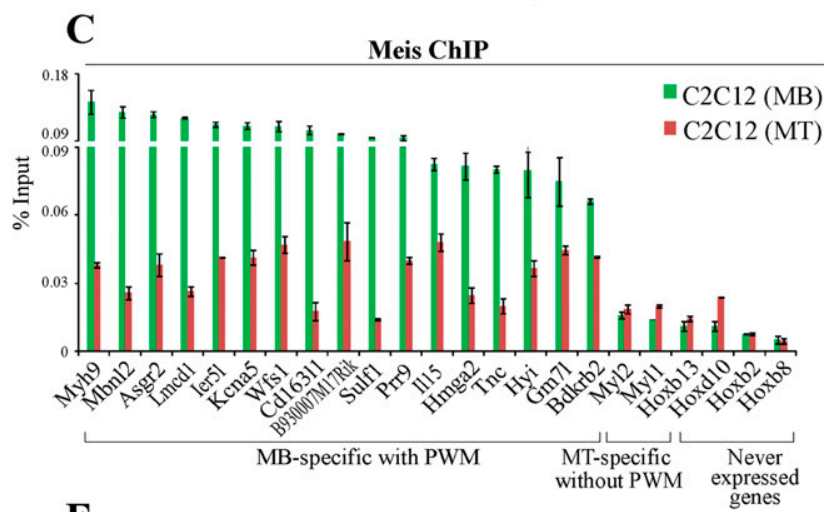

$\mathbf{E}$

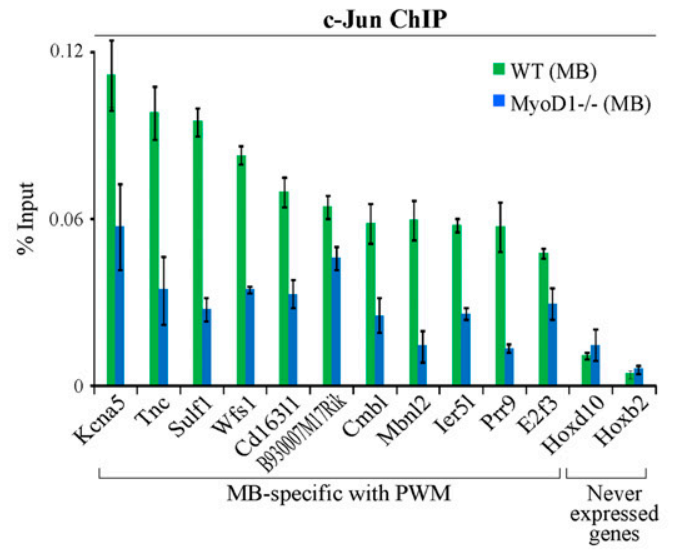

G

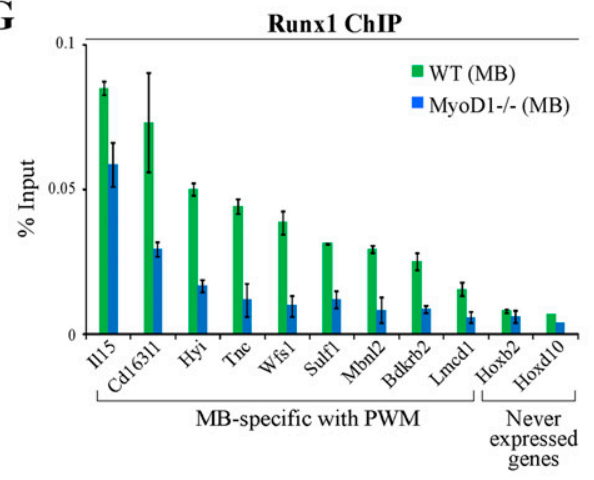

Figure 6. Condition-specific and MyoD1-dependent binding of transcription factors to MyoD1-bound enhancers. $(A-D) \mathrm{qChIP}$ was performed to detect binding of c-Jun $(A)$, Jdp2 $(B)$, Meis $(C)$, and Runx1 $(D)$ in C2C12 myoblasts and myotubes. qChIP analysis using antibodies against E2F4 confirmed the specificity of our binding events. (E-G) qChIP indicated that MyoD1 ablation results in reduced recruitment of c-Jun $(E)$, Jdp2 $(F)$, and Runx1 $(G)$. We included several negative controls corresponding to myotube-specific enhancers that are not enriched for the predicted binding motif and genomic regions located upstream of genes that were never expressed. 
A

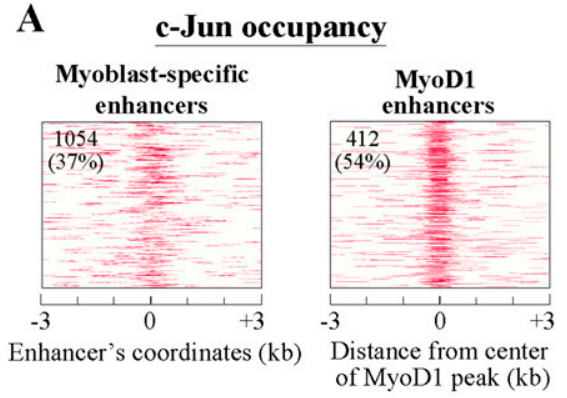

B

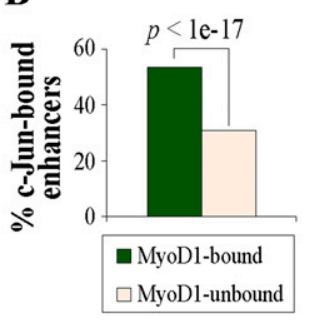

C

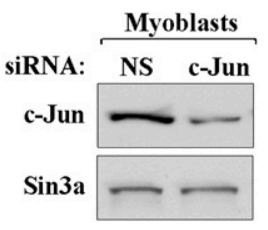

D

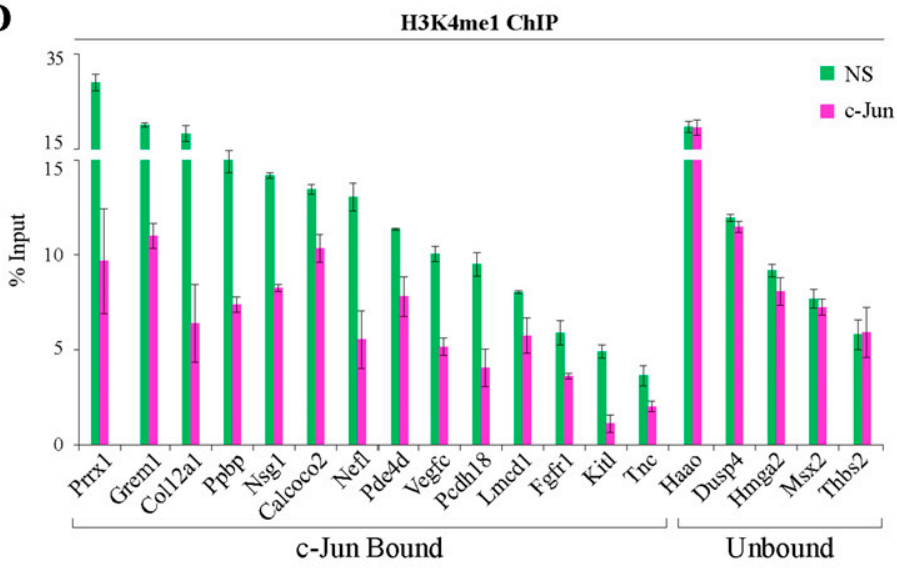

$\mathbf{E}$

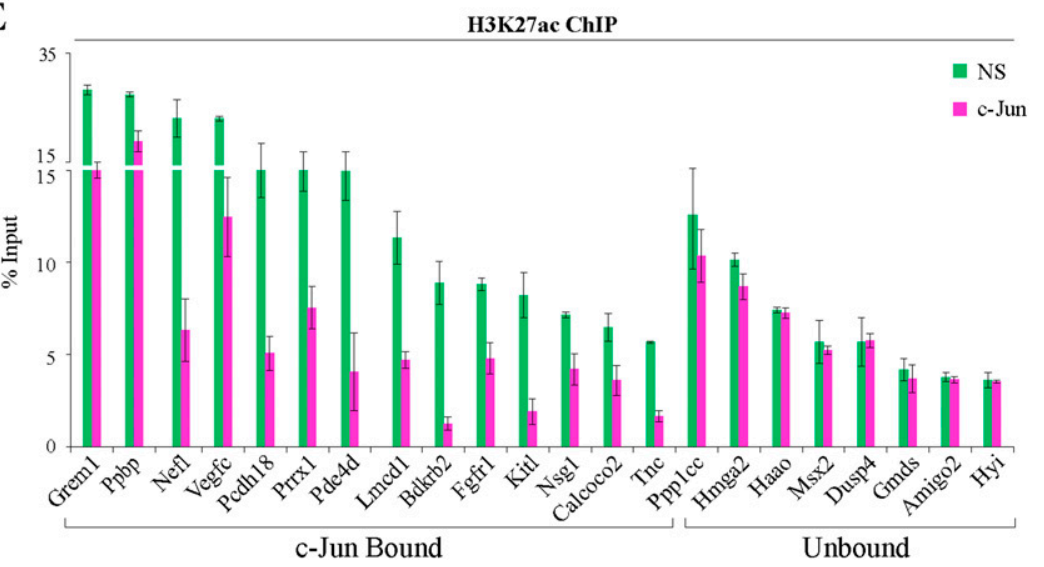

Figure 7. c-Jun loss results in reduced assembly of c-Jun-bound enhancers. (A) Maps of myoblast-specific enhancers that bind MyoD1 and c-Jun within $\pm 3 \mathrm{~kb}$ of the center of myoblast-specific enhancers. The number and percentage of condition-specific enhancers significantly enriched with c-Jun are indicated in the top left corner of each map. $(B)$ Recruitment of c-Jun is significantly higher on MyoD1-bound enhancers. $(C)$ Western blot detection of c-Jun in whole-cell extracts prepared from growing C2C12 myoblasts $48 \mathrm{~h}$ after transfection with an siRNA pool targeting c-Jun or with a control, nonsilencing siRNA (NS). $(D, E)$ qChIP was performed to detect enhancer-related marks-H3K4me1 $(D)$ and $\mathrm{H} 3 \mathrm{~K} 27 \mathrm{ac}(E)$, as indicated-on myoblast-specific enhancers bound by c-Jun after treatment of myoblasts with c-Jun and control siRNAs. Several myoblast-specific enhancers not bound by c-Jun were tested as negative controls and showed insignificant alterations in the levels of all marks tested.

miss the appropriate window necessary for the "prepatterning" of myoblast enhancers.

\section{Discussion}

\section{Identification of enhancer elements in skeletal muscle}

Here, we identified a compendium of enhancers that function in mammalian skeletal muscle, providing a rich source of data for future discovery of enhancer-associated mechanisms.

Our enhancer identification is based on several lines of evidence. First, we showed that each region is characterized by a set of features known to be associated with enhancers. Second, we showed that distal elements with associated condition-specific signatures are linked to adjacent genes expressed at appropriate times. Third, known 
A

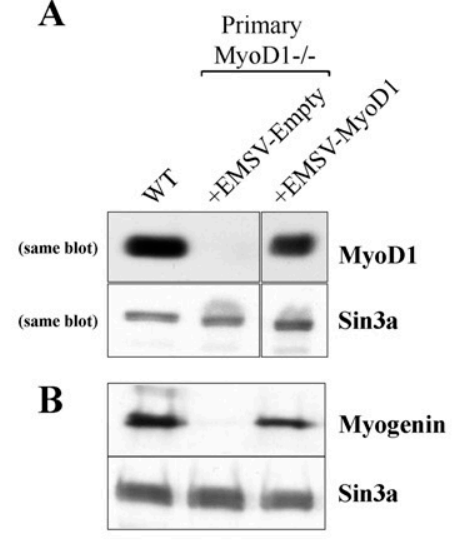

C

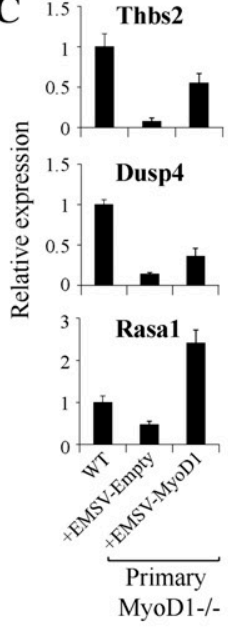

D

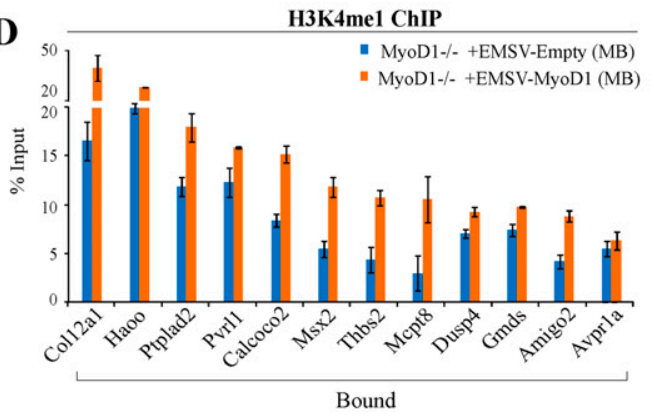

E

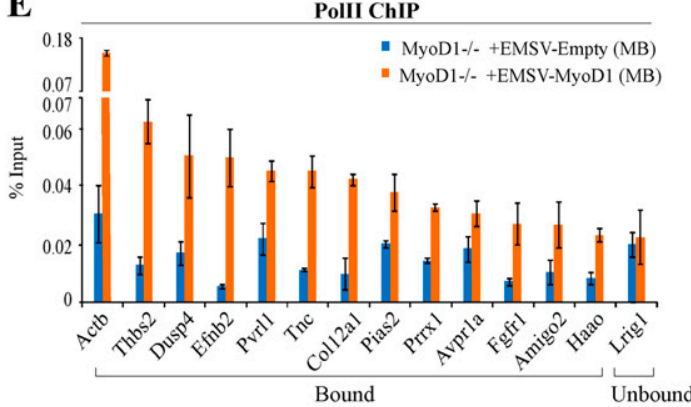

G

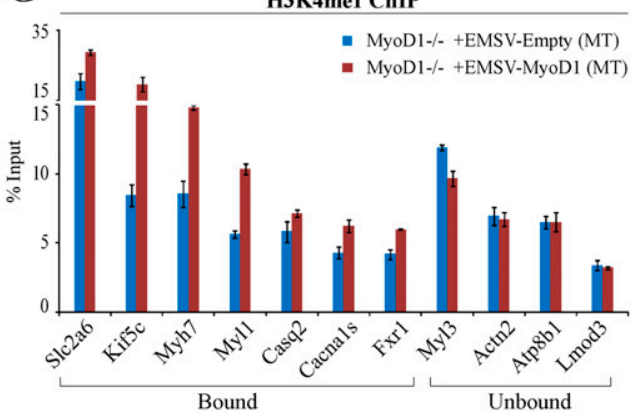

H H3K27ac ChIP

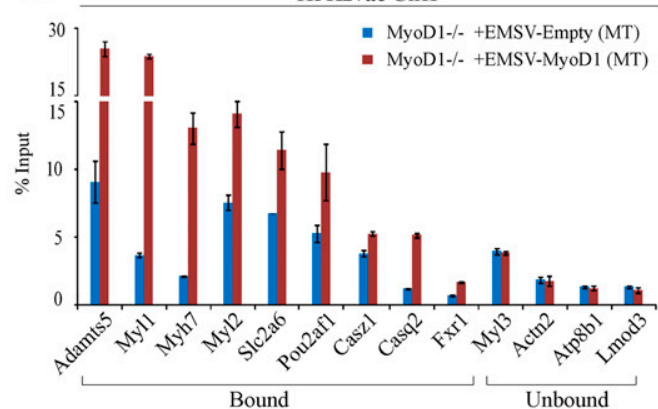

I

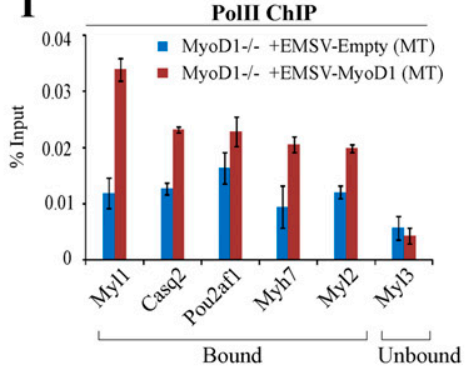

F

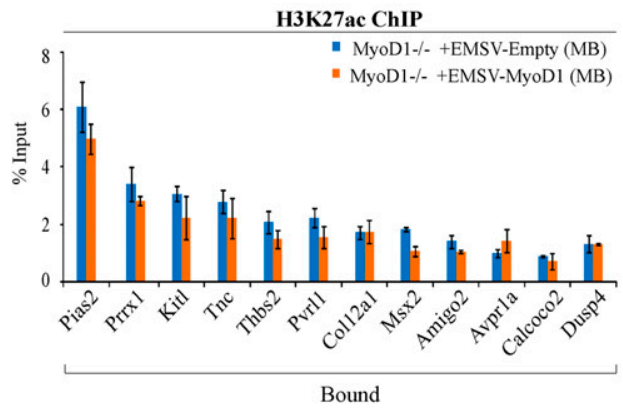

J
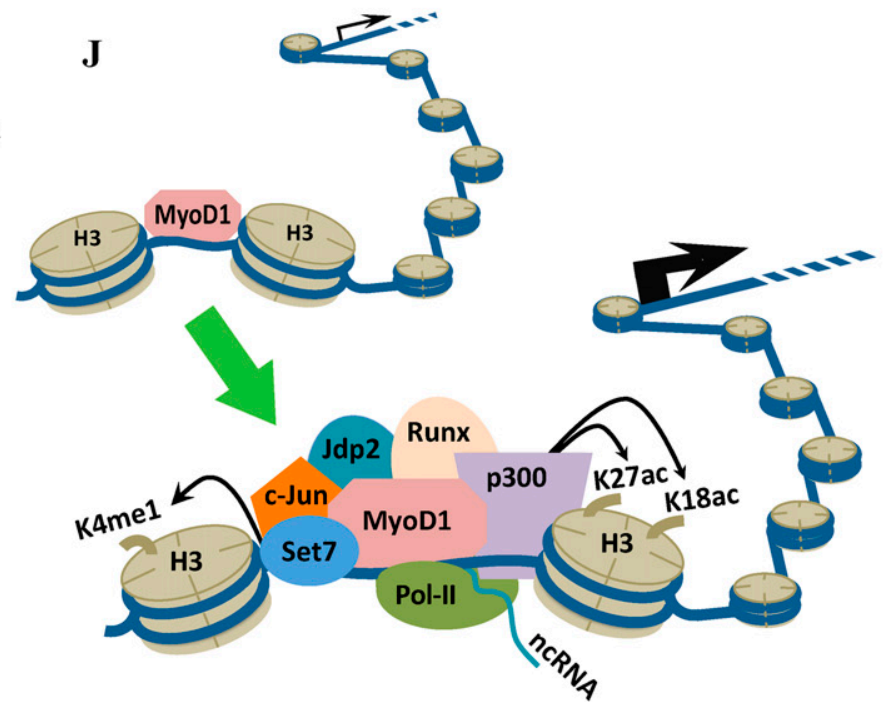

Figure 8. Exogenous MyoD1 expression restores enhancer assembly in primary MyoD1 ${ }^{-/-}$myoblasts. $(A)$ Western blot detection of MyoD1 in nuclear extracts prepared from growing wild-type, $M y o D 1^{-1-}$, and $M y o D 1^{-/-}$(rescued) primary myoblasts that stably express exogenous MyoD1. Sin3A is shown as a loading control. $(B)$ Western blotting of nuclear extracts of confluent myoblasts indicates that myogenin expression is restored to levels comparable with those of wild-type cells. (C) RT-qPCR analysis of three target genes associated with myoblast-specific enhancers bound by MyoD1. We note that each of the promoters associated with these genes was devoid of MyoD1 binding. $(D-F)$ qChIP analysis was carried out to determine the impact of MyoD1 reconstitution in primary myoblasts on deposition of enhancer-related marks H3K4me1 $(D)$, Pol II $(E)$, and H3K27ac $(F)$. (G-I) qChIP analysis for H3K4me1 $(G)$, H3K27ac $(H)$, and Pol II $(I)$ was performed to examine indicated marks on myotube-specific enhancers after MyoD1 expression. Error bars for all qChIP and RT-qPCR data represent standard error of the mean (SEM). (J) Model in which MyoD1 acts to assemble enhancers in muscle. Interactions between MyoD1 and other factors are essential for enhancer assembly and acquisition of a transcriptionally active state. See the text for details. 
enhancer regions are recovered in our data set. Fourth, we showed that MyoD1 and a cohort of TFs co-occupy these distal elements. We showed that these enhancers may function to augment transcription of thousands of proteincoding genes and identified a large number of ncRNAs that are specifically associated with these enhancers.

Moreover, the ability to recreate enhancer assembly through re-expression of a key regulator, MyoD1, offers a robust and well-defined system in which to explore the step-wise assembly of enhancers. As expected from previous genome-scale studies, we found that the epigenetic signatures associated with muscle enhancers were unique to this tissue, although to some extent they resembled the pattern observed in adipocytes, in line with the fact that skeletal muscle cells and adipocytes are derived from a common MSC precursor. Interestingly, we noted that H3K27ac enrichment over the centers of putative enhancer regions was more diffuse in adipocytes than the corresponding regions in muscle, suggesting that mechanisms that regulate its deposition and enhancer activity may differ to some extent in the two tissues. It will be interesting to obtain corresponding chromatin state maps for MSCs and satellite cells and compare those maps with the committed lineages presented here.

We found evidence that many genes expressed at higher levels in myotubes were linked to associated enhancers showing constitutive modifications in both states, suggesting that enhancers could be "primed," awaiting expression of specific transcriptional regulators later in the differentiation program. We also discovered that enhancer association with conditionally expressed genes decreased as a function of increasing distance from the TSS (Supplemental Fig. S1G). Such increased distance could impose a functional barrier that limits the ability of enhancers to transmit their signal. A looping model for enhancer function has been proposed, and this observation could support the notion that short loops may have a stronger positive impact on gene transcription than longer loops (Blackwood and Kadonaga 1998). Moreover, we found that the distance between enhancers and their nearest linked genes decreased as myoblasts differentiated into myotubes, suggesting that chromatin could undergo conformational changes in the differentiated state that facilitate and augment enhancer activity.

It is likely that MyoD1 potentiates expression of linked protein-coding genes by binding to the promoters of these genes, in addition to exerting an enhancer assembly function at a distance, as described here. Nevertheless, an analysis of linked promoters (defined by $\pm 1 \mathrm{~kb}$ around the TSS) showed that for genes associated with MyoD1bound enhancers, the majority of these linked promoters ( $\sim 60 \%$ of linked promoters in either myoblasts or myotubes) is not occupied by MyoD1 in vivo. Thus, in the absence of MyoD1, the expression of these genes is likely to be altered as result of deregulation of enhancer assembly (orchestrated by MyoD1) rather than through deregulation of promoters. In addition, many of the enhancers that were examined in our study are linked to promoters that do not exhibit MyoD1 occupancy in vivo (Fig. 8C; detailed in Supplemental Table S4), suggesting that expression of these genes was mediated primarily through MyoD1 binding to enhancers. We further note that in many cases where MyoD1 is recruited to both the promoter and enhancer, the signal detected at the promoter could result from looping, a mechanism associated with enhancer-mediated, long-distance gene activation.

\section{A pivotal role for MyoD1 in enhancer assembly}

In this study, we provide new information on the role of MyoD1 in differentiation by demonstrating an unequivocal role for this factor in the assembly of active enhancers. These studies dramatically extend previous work in which MyoD1-binding sites were discovered genomewide (Blais et al. 2005; Cao et al. 2010). More recently, Soleimani et al. (2012) identified a set of MyoD1-bound peaks, a substantial number of which were intergenic and $\sim 5600$ of which overlapped with Cao et al. (2010). Overall, we found that while the majority of MyoD1binding sites is located within gene-associated or previously uncharacterized intergenic regions (Cao et al. 2010), only $\sim 10 \%$ of all MyoD1-binding sites were located within active muscle enhancers (in both myoblasts and myotubes), suggesting that there is highly selective binding of MyoD1 to chromatin at transcriptional enhancing regions.

Our experiments with $\mathrm{C} 2 \mathrm{C} 12$ and primary skeletal muscle cells led us to several important conclusions. First, depletion of MyoD1 results in a dramatic alteration of enhancer signatures, typified by the loss of H3K4me1 and H3K27ac as well as p300 and Pol II recruitment. Second, we show that the loss of Set7, an H3K4 monomethylase, could explain the observed reductions in H3K4me1 in $\mathrm{MyoD}^{-1-}$ cells. Third, we found that c-Jun, Jdp2, and Runxl were recruited to enhancer regions in a spatially constrained domain centered around MyoD1, and, strikingly, each of these factors was recruited in a MyoD1-dependent manner. Collectively, these data suggest that MyoD1 assists in the corecruitment of several sequence-specific TFs, Set7, and p300 at enhancers (Fig. 8J). As a result of Set 7 and p300 recruitment, H3K4 and H3K27 are monomethylated and acetylated, respectively. Fourth, depletion of MyoD1 resulted in significant alterations in the expression of ncRNAs associated with enhancers. To our knowledge, this is the first indication that MyoD1 may stimulate expression of ncRNAs at enhancers. Fifth, we found that MyoD1 re-expression in primary MyoD1null myoblasts rescued some (H3K4me1 and Pol II) but not all $(\mathrm{H} 3 \mathrm{~K} 27 \mathrm{ac})$ hallmarks of these enhancers, whereas H3K27ac was also restored in myotubes. These observations indicate that the timely function of MyoD1 is essential for the correct assembly of muscle enhancers. A failure to express MyoD1 within an essential period would result in inefficient recruitment of enhancerpromoting factors and incomplete assembly of conditionspecific enhancers. In addition, it is likely that chromatin plasticity at enhancer regions is temporally limited and irreversibly constrained once the appropriate window for enhancer assembly has passed. 
While further studies will be required to address this condition-dependent restoration of chromatin signatures at enhancers, this finding suggests that any "code" associated with enhancers may need to be refined or augmented by additional factors, such as TF-binding events or other stimuli, in a condition-specific manner. Our studies set the stage for exciting future experiments aimed at dissecting the nature of muscle-specific enhancers.

In addition, it has been proposed that MyoD1 deficiency results in an elevated self-renewal rate, increasing the number of precursor (satellite) cells and limiting progression through the myogenic program (Megeney et al. 1996). In this regard, we speculate that MyoD1 could act to remodel and facilitate assembly of muscle enhancers, thereby diverting cells away from nonmuscle lineages.

Our study identified the critical genomic regulatory regions that underlie the network that orchestrates the myogenic program. It will be interesting to align these loci with maps of single-nucleotide polymorphisms (SNPs) and mutations linked to genetic disorders of skeletal muscle, since aberrations that may occur within these newly identified myogenic enhancers could impair the regulation of target genes essential for muscle structure and function. Ultimately, our study could shed new light on pathological mechanisms initiated by misregulation of these critical regulatory regions.

\section{Materials and methods}

\section{Quantitative RT-PCR, transfections, and protein detection}

RNA isolation, reverse transcription, and real-time quantitative PCR were performed as described (Asp et al. 2011). In each case, expression was normalized to a control gene (Sparc) that is invariant throughout differentiation. All primer sequences are listed in Supplemental Table S8. Transfections were performed in myoblasts using Lipofectamine LTX (Invitrogen). Nuclear extracts were prepared as described (Asp et al. 2009), with the modification that hypotonic homogenization buffer was adjusted to $10 \mathrm{mM} \mathrm{KCl}$, and nuclear extraction buffer was adjusted to $0.42 \mathrm{M} \mathrm{KCl}$.

\section{Antibodies, ChIP, and ChIP-seq}

ChIP was performed as described (Blais et al. 2007) using antibodies listed in the Supplemental Material. ChIP experiments to detect p300 enrichment in primary myoblasts were performed by fixing cells for $5 \mathrm{~min}$ in $1.5 \mathrm{mM}$ ethylene glycol-bis succinimidylsuccinate (EGS) and subsequent fixation in $1 \%$ formaldehyde at room temperature for $10 \mathrm{~min}$. To quantitatively measure changes in histone marks and factors, the linear range for ChIP and ChIP-seq experiments was empirically determined using serial titrations of antibody and chromatin and then plotting enrichment as a function of chromatin amount. All ChIP experiments were carried out using optimized antibody/chromatin ratios. ChIP-seq experiments were performed as described (Asp et al. 2011).

\section{Analysis of ChIP-seq data and enhancer identification}

ChIP-seq data were analyzed as described previously (Asp et al. 2011), with the modifications noted in the Supplemental Material. Enhancers were identified as described in the Supplemental Material.

\section{Acknowledgments}

We thank P. Asp for assistance with initial ChIP-seq experiments. We are grateful to the members of the Dynlacht laboratory for advice and assistance and acknowledge the New York University Genome Technology Core. We thank V. Soleimani for critical reading of our manuscript; M. Frith, I. Barozzi, and D. Cittaro for their assistance with CLOVER; and R. Bonasio for help with Bowtie. This work was supported by grants to B.D.D. (NIH CA077245 and R01GM067132) and NIA AG040894 to C.B.

\section{References}

Asp P, Acosta-Alvear D, Tsikitis M, van Oevelen C, Dynlacht BD. 2009. E2f3b plays an essential role in myogenic differentiation through isoform-specific gene regulation. Genes Dev 23: 37-53.

Asp P, Blum R, Vethantham V, Parisi F, Micsinai M, Cheng J, Bowman C, Kluger Y, Dynlacht BD. 2011. Genome-wide remodeling of the epigenetic landscape during myogenic differentiation. Proc Natl Acad Sci 108: E149-E158. doi: 10.1073/ pnas.1102223108.

Bengal E, Ransone L, Scharfmann R, Dwarki VJ, Tapscott SJ, Weintraub H, Verma IM. 1992. Functional antagonism between c-Jun and MyoD proteins: A direct physical association. Cell 68: 507-519.

Blackwood EM, Kadonaga JT. 1998. Going the distance: A current view of enhancer action. Science 281: 60-63.

Blais A, Tsikitis M, Acosta-Alvear D, Sharan R, Kluger Y, Dynlacht BD. 2005. An initial blueprint for myogenic differentiation. Genes Dev 19: 553-569.

Blais A, van Oevelen CI, Margueron R, Acosta-Alvear D, Dynlacht BD. 2007. Retinoblastoma tumor suppressor protein-dependent methylation of histone $\mathrm{H} 3$ lysine 27 is associated with irreversible cell cycle exit. I Cell Biol 179: 1399-1412.

Bulger M, Groudine M. 2011. Functional and mechanistic diversity of distal transcription enhancers. Cell 144: 327339.

Cabili MN, Trapnell C, Goff L, Koziol M, Tazon-Vega B, Regev A, Rinn JL. 2011. Integrative annotation of human large intergenic noncoding RNAs reveals global properties and specific subclasses. Genes Dev 25: 1915-1927.

Cao Y, Kumar RM, Penn BH, Berkes CA, Kooperberg C, Boyer LA, Young RA, Tapscott SJ. 2006. Global and gene-specific analyses show distinct roles for Myod and Myog at a common set of promoters. EMBO I 25: 502-511.

Cao Y, Yao Z, Sarkar D, Lawrence M, Sanchez GJ, Parker MH, MacQuarrie KL, Davison J, Morgan MT, Ruzzo WL, et al. 2010. Genome-wide MyoD binding in skeletal muscle cells: A potential for broad cellular reprogramming. Dev Cell 18: 662-674.

Creyghton MP, Cheng AW, Welstead GG, Kooistra T, Carey BW, Steine EJ, Hanna J, Lodato MA, Frampton GM, Sharp PA, et al. 2010. Histone H3K27ac separates active from poised enhancers and predicts developmental state. Proc Natl Acad Sci 107: 21931-21936.

De Santa F, Barozzi I, Mietton F, Ghisletti S, Polletti S, Tusi BK, Muller H, Ragoussis J, Wei CL, Natoli G. 2010. A large fraction of extragenic RNA pol II transcription sites overlap enhancers. PLOS Biol 8: e1000384. doi: 10.1371/journal. pbio. 1000384.

Frith MC, Fu Y, Yu L, Chen JF, Hansen U, Weng Z. 2004. Detection of functional DNA motifs via statistical overrepresentation. Nucleic Acids Res 32: 1372-1381. 
Gerber AN, Klesert TR, Bergstrom DA, Tapscott SJ. 1997. Two domains of MyoD mediate transcriptional activation of genes in repressive chromatin: A mechanism for lineage determination in myogenesis. Genes Dev 11: 436-450.

Guttman M, Amit I, Garber M, French C, Lin MF, Feldser D, Huarte M, Zuk O, Carey BW, Cassady JP, et al. 2009. Chromatin signature reveals over a thousand highly conserved large non-coding RNAs in mammals. Nature 458: 223-227.

Guttman M, Donaghey J, Carey BW, Garber M, Grenier JK, Munson G, Young G, Lucas AB, Ach R, Bruhn L, et al. 2011. lincRNAs act in the circuitry controlling pluripotency and differentiation. Nature 477: 295-300.

He A, Kong SW, Ma Q, Pu WT. 2011. Co-occupancy by multiple cardiac transcription factors identifies transcriptional enhancers active in heart. Proc Natl Acad Sci 108: 5632-5637.

Heintzman ND, Stuart RK, Hon G, Fu Y, Ching CW, Hawkins RD, Barrera LO, Van Calcar S, Qu C, Ching KA, et al. 2007. Distinct and predictive chromatin signatures of transcriptional promoters and enhancers in the human genome. Nat Genet 39: 311-318.

Heintzman ND, Hon GC, Hawkins RD, Kheradpour P, Stark A, Harp LF, Ye Z, Lee LK, Stuart RK, Ching CW, et al. 2009. Histone modifications at human enhancers reflect global cell-type-specific gene expression. Nature 459: 108-112.

Jin Q, Yu LR, Wang L, Zhang Z, Kasper LH, Lee JE, Wang C, Brindle PK, Dent SY, Ge K. 2011. Distinct roles of GCN5/ PCAF-mediated H3K9ac and CBP/p300-mediated H3K18/ $27 \mathrm{ac}$ in nuclear receptor transactivation. EMBO J 30: 249262.

Kim TK, Hemberg M, Gray JM, Costa AM, Bear DM, Wu J, Harmin DA, Laptewicz M, Barbara-Haley K, Kuersten S, et al. 2010. Widespread transcription at neuronal activity-regulated enhancers. Nature 465: 182-187.

Liu Y, Chu A, Chakroun I, Islam U, Blais A. 2010. Cooperation between myogenic regulatory factors and SIX family transcription factors is important for myoblast differentiation. Nucleic Acids Res 38: 6857-6871.

Megeney LA, Kablar B, Garrett K, Anderson JE, Rudnicki MA. 1996. MyoD is required for myogenic stem cell function in adult skeletal muscle. Genes Dev 10: 1173-1183.

Mikkelsen TS, Xu Z, Zhang X, Wang L, Gimble JM, Lander ES, Rosen ED. 2010. Comparative epigenomic analysis of murine and human adipogenesis. Cell 143: 156-169.

Ponting CP. 2008. The functional repertoires of metazoan genomes. Nat Rev Genet 9: 689-698.

Prabhakar S, Poulin F, Shoukry M, Afzal V, Rubin EM, Couronne O, Pennacchio LA. 2006. Close sequence comparisons are sufficient to identify human cis-regulatory elements. Genome Res 16: 855-863.

Rada-Iglesias A, Bajpai R, Swigut T, Brugmann SA, Flynn RA, Wysocka J. 2011. A unique chromatin signature uncovers early developmental enhancers in humans. Nature 470: 279283.

Rudnicki MA, Braun T, Hinuma S, Jaenisch R. 1992. Inactivation of MyoD in mice leads to up-regulation of the myogenic HLH gene Myf-5 and results in apparently normal muscle development. Cell 71: 383-390.

Sassoon D, Lyons G, Wright WE, Lin V, Lassar A, Weintraub H, Buckingham M. 1989. Expression of two myogenic regulatory factors myogenin and MyoD1 during mouse embryogenesis. Nature 341: 303-307.

Seale P, Bjork B, Yang W, Kajimura S, Chin S, Kuang S, Scime A, Devarakonda S, Conroe HM, Erdjument-Bromage H, et al. 2008. PRDM16 controls a brown fat/skeletal muscle switch. Nature 454: 961-967.
Siepel A, Bejerano G, Pedersen JS, Hinrichs AS, Hou M, Rosenbloom K, Clawson H, Spieth J, Hillier LW, Richards S, et al. 2005. Evolutionarily conserved elements in vertebrate, insect, worm, and yeast genomes. Genome Res 15: 10341050.

Soleimani VD, Yin H, Jahani-Asl A, Ming H, Kockx CE, van Ijcken WF, Grosveld F, Rudnicki MA. 2012. Snail regulates MyoD binding-site occupancy to direct enhancer switching and differentiation-specific transcription in myogenesis. Mol Cell 47: 457-468.

Tao Y, Neppl RL, Huang ZP, Chen J, Tang RH, Cao R, Zhang Y, Jin SW, Wang DZ. 2011. The histone methyltransferase Set7/ 9 promotes myoblast differentiation and myofibril assembly. J Cell Biol 194: 551-565.

Trapnell C, Williams BA, Pertea G, Mortazavi A, Kwan G, van Baren MJ, Salzberg SL, Wold BJ, Pachter L. 2010. Transcript assembly and quantification by RNA-Seq reveals unannotated transcripts and isoform switching during cell differentiation. Nat Biotechnol 28: 511-515.

Vernimmen D, Lynch MD, De Gobbi M, Garrick D, Sharpe JA, Sloane-Stanley JA, Smith AJ, Higgs DR. 2011. Polycomb eviction as a new distant enhancer function. Genes Dev 25: 1583-1588.

Visel A, Minovitsky S, Dubchak I, Pennacchio LA. 2007. VISTA enhancer browser-a database of tissue-specific human enhancers. Nucleic Acids Res 35: D88-D92. doi: 10.1093/nar/ gk1822.

Visel A, Prabhakar S, Akiyama JA, Shoukry M, Lewis KD, Holt A, Plajzer-Frick I, Afzal V, Rubin EM, Pennacchio LA. 2008. Ultraconservation identifies a small subset of extremely constrained developmental enhancers. Nat Genet 40: 158-160.

Visel A, Blow MJ, Li Z, Zhang T, Akiyama JA, Holt A, PlajzerFrick I, Shoukry M, Wright C, Chen F, et al. 2009. ChIP-seq accurately predicts tissue-specific activity of enhancers. $\mathrm{Na}$ ture 457: 854-858.

Wang Z, Zang C, Rosenfeld JA, Schones DE, Barski A, Cuddapah S, Cui K, Roh TY, Peng W, Zhang MQ, et al. 2008. Combinatorial patterns of histone acetylations and methylations in the human genome. Nat Genet 40: 897-903.

Yuan W, Condorelli G, Caruso M, Felsani A, Giordano A. 1996. Human p300 protein is a coactivator for the transcription factor MyoD. J Biol Chem 271: 9009-9013. 


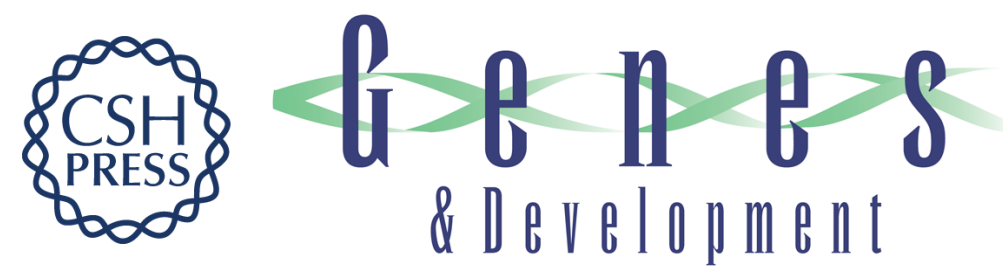

\section{Genome-wide identification of enhancers in skeletal muscle: the role of MyoD1}

Roy Blum, Vasupradha Vethantham, Christopher Bowman, et al.

Genes Dev. 2012, 26:

Access the most recent version at doi:10.1101/gad.200113.112

Supplemental
Material http://genesdev.cshlp.org/content/suppl/2012/12/18/26.24.2763.DC1

References This article cites 40 articles, 16 of which can be accessed free at:

http://genesdev.cshlp.org/content/26/24/2763.full.html\#ref-list-1

License

Email Alerting

Receive free email alerts when new articles cite this article - sign up in the box at the top

Service

right corner of the article or click here.

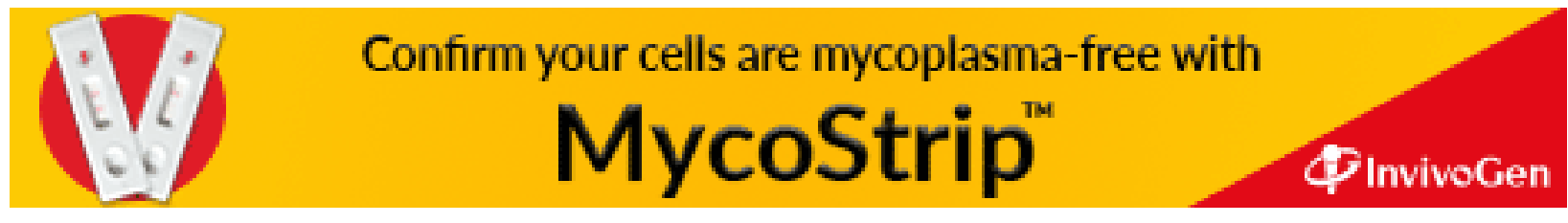

